\begin{tabular}{c|c|c|} 
SELECCIONES MATEMÁTICAS \\
Universidad Nacional de Trujillo \\
ISSN: $2411-1783$ (Online) \\
2021; Vol. 8(1): 196-217.
\end{tabular}

MATHEMATICS EDUCATION

\title{
Some Ideas on the Evolution of Real Mathematical Analysis
}

\section{Algunas Ideas Sobre la Evolución del Análisis Matemático Real}

Alejandro Ortiz Fernández $(\mathbb{D}$

Received, Feb. 28, 2021

Accepted, Jun. 30, 2021

How to cite this article:

Ortíz A. Some Ideas on the Evolution of Real Mathematical Analysis. Selecciones Matemáticas. 2021;8(1):196-217. http://dx.doi.org/10.17268/sel.mat.2021.01.17

\begin{abstract}
From Antiquity to the 19th century, we give some brief comments and historical examples that motivate and illustrate some results in the evolution of mathematical analysis.
\end{abstract}

Keywords . Archimedes, Newton, Euler, Fourier, Riemann, Cantor.

\section{Resumen}

De la antigüedad al siglo XIX, damos algunos breves comentarios y ejemplos históricos que nos motivan e ilustran sobre algunos resultados en la evolución del análisis matemático.

Palabras clave. Arquímedes, Newton, Euler, Fourier, Riemann, Cantor.

1. Los Infinitesimales. Orígenes del Análisis Infinitesimal. Retrocedamos hasta la época de los pitagóricos, época en que se trató de explicar la naturaleza de $\sqrt{2}$ y del primer encuentro con el infinito. Este problema condujo a tratar con cantidades muy pequeñas y así los números naturales $1,2,3$, ... no servían para explicar esta cuestión: medir la diagonal del cuadrado de lado 1, y otras situaciones que surgieron. En otras palabras, "es imposible encontrar dos números enteros tales que el cuadrado de uno sea igual al doble del cuadrado del otro". Aún, "la razón del lado de un cuadrado y su diagonal no puede expresarse vía la razón de dos números enteros". Así surgió una profunda crisis en la Antigüedad, la que habría de durar alrededor de dos mil años. De esta manera, desde entonces los matemáticos tuvieron que pensar y enfrentar a los "infinitesimales", a las cantidades inconmensurables.

Fue el matemático Eudoxo de Cnido quien enfrentó los momentos críticos causados por el infinito y por las paradojas surgidas. Su teoría de las proposiciones está relacionado con el método de exhaución, expuesto en el libro V de Euclides. En las ideas de Eudoxo están los gérmenes de los números irracionales, fundamento del análisis moderno. Además, la manera de hallar área, volúmenes y centros de gravedad de figuras geométricas están dentro de lo que sería el cálculo integral. Arquímedes, gran matemático, fue un continuador de las ideas de Eudoxo, las perfecciona y establece con rigor los infinitesimales, una idea que acompaña a los matemáticos por siglos. En el ejemplo 1, [6], se presenta como Arquímedes encuentra el área de un sector parabólico: como el límite de áreas de figuras $P_{n}$, donde $P_{n}$ es un polígono cuya área es conocida.

En el argumento dado, está una progresión geométrica $1, a, a^{2}, \ldots a^{n-1}$, cuya suma es igual a $\frac{1-a^{n}}{1-a}, a \neq$

1. En tal ejemplo, $a=\frac{1}{4}$. Hoy sabemos que si $|a|<1$, se tiene que $1+a+a^{2}+a^{3}+\ldots+a^{n}+\ldots=\frac{1}{1-a}$. Así Arquímedes logra establecer que

$$
1+\frac{1}{4}+\frac{1}{4^{2}}+\frac{1}{4^{3}}+\ldots=\frac{1}{1-\frac{1}{4}}=\frac{4}{3} .
$$

*Sección Matemática. Pontificia Universidad Católica del Perú, Lima, Perú. (jorti z @pucp. edu • pe). 


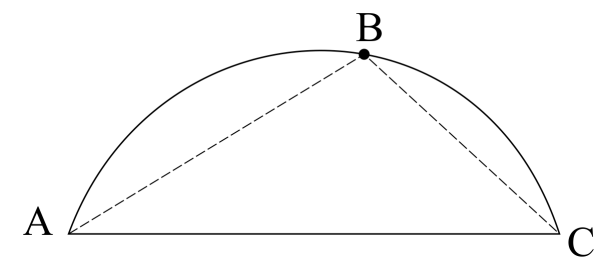

Figura 1.1: Inicio de la cuadratura de la parábola

Así se tiene:

Teorema 1.1 (Arquímedes).

Sea $B$ el vértice de un segmento parabólico con base $A C$.

Entonces, área del segmento $A B C=\frac{4}{3}$ área triangular $A B C$.

En [4], pag. cxlii, podemos ver un conjunto de resultados en donde apreciamos resultados que se anticipan al cálculo integral. También en el tratado sobre las Espirales como en el de las Conoides y Esferoides, Arquímedes llega a fórmulas equivalentes a nuestras conocidas fórmulas

$$
\int_{0}^{c} x d x=\frac{1}{2} c^{2} \text { y } \int_{0}^{c} x^{2} d x=\frac{1}{3} x^{3}
$$

(ver, por ejemplo, la pag. cxlviii del citado libro para los detalles). Debemos remarcar que en su Tratado sobre las Espirales, Arquímedes investiga el trazado de tangentes a las espirales y de esta manera es también un precursor del cálculo diferencial. Como apreciamos, es grande las contribuciones de este genio griego en la determinación de áreas y volúmenes del círculo, de la esfera, de cilindros y de muchos otros resultados, muchos de ellos se han perdido. El gran mérito de los matemáticos griegos fue el inicio del quehacer con el "infinito" y de pensar en los "infinitesimales", ideas profundas que mantuvieron ocupados a filósofos y matemáticos por siglos. Arquímedes fue genial como matemático puro y como matemático aplicado, le debemos muchas obras maestras; en [4] podemos apreciar lo abundante de su producción. Como ejemplos veamos su investigación del número "pi”, $\pi$, y hallar el área de un círculo.

Ejemplo 1.1 (Arquímedes. área del Círculo. $\pi=$ ?).

En la historia de la matemática los números $\pi, i$, e juegan un importante rol. En la antigua Grecia, Hipócrates y Eudoxo meditaron la siguiente situación: "en un círculo inscribieron polígonos, cada vez con un mayor número de lados”, una idea que se remonta a la época de Sócrates. Euclides, en sus elementos, también reflexiona en esta cuestión, ¿qué pasa si seguimos inscribiendo polígonos? La intuición entra en juego; Euclides sugiere usar la idea de "límite" como un paso fundamental para obtener el área del círculo. El paso siguiente fue dado por Arquímedes usando el método de exhaución. Ver [6], ejemplo 1. En este panorama surge $\pi$, un número maravilloso! Arquímedes organizó una cacería de $\pi$. Veamos

Actualmente sabemos que la razón de la longitud de una circunferencia con la longitud del diámetro respectivo es la misma para cualquier circunferencia.

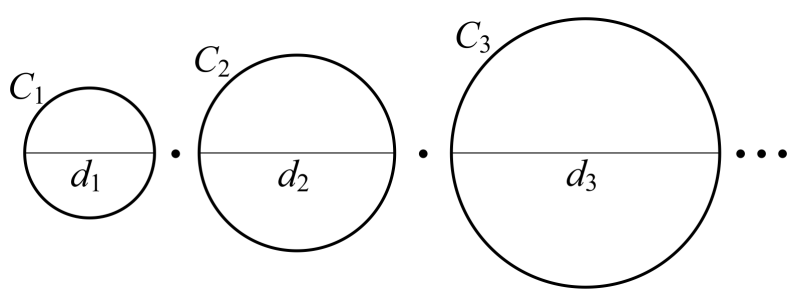

Figura 1.2: Definición de $\pi$

Así,

$$
\frac{C_{1}}{D_{1}}=\frac{C_{2}}{D_{2}}=\frac{C_{3}}{D_{3}}=\frac{C_{4}}{D_{4}}=\ldots=\text { constante } \equiv k
$$

Por definición, $\pi=k$. En general, $\pi=\frac{C}{D}$ ó $C=\pi D$.

Desde la época de la Antigüedad, en particular en el período de los griegos, el cálculo del valor de $\pi$ fue un reto a la inteligencia humana. Arquímedes atacó este problema con mucha imaginación. En su libro "De la medida del círculo" nos dice que la razón de la circunferencia al diámetro está comprendida entre $3 \frac{10}{71}$ y $3 \frac{1}{7}$.

También nos dice que la razón (del área) del círculo con el cuadrado (de la longitud) del diámetro es 11:14. 
Veamos algunos argumentos. El libro XII de Euclides contiene la:

Proposición XII.2. Círculos son uno a otro como los cuadrados de sus diámetros.

Observación 1.1. Este resultado fue usado por Hipócrates en la cuadratura de la luna.

En efecto, sea $C_{1}$ una circunferencia, $A_{1}$ el área del círculo respectivo y $D_{1}$ es su diámetro. Similar con la circunferencia $C_{2}$. Entonces tenemos $(R=$ radio $)$

esto es, $\frac{A_{1}}{D_{1}^{2}}=\frac{A_{2}}{D_{2}^{2}}=$ constante $=k_{1}$.

$$
\frac{A_{1}}{A_{2}}=\frac{\pi R_{1}^{2}}{\pi R_{2}^{2}}=\frac{\left(2 R_{1}\right)^{2}}{\left(2 R_{2}\right)^{2}}=\frac{D_{1}^{2}}{D_{2}^{2}}
$$

En general, $\frac{A}{D^{2}}=k_{1}$. ¿Existe alguna conexión entre las constantes $k$ (uni-dimensional) y la constante $k_{1}$ (bi-dimensional)? Parece que Euclides no encontró tal conexión. En el libro citado, Arquímedes probó la fórmula para encontrar el área circular en función de $\pi$, estableciendo un puente crítico entre la circunferencia y el área del círculo. En esta dirección veamos algunas ideas preliminares. Consideremos un polígono regular con centro $O$, perímetro $P$ y apotema $a$. Se tiene el

Teorema 1.2. El área de un polígono regular es $\frac{1}{2} a P$.

Demostración:

Supongamos que el polígono tuviera $n$ lados, cada uno de longitud $b$.

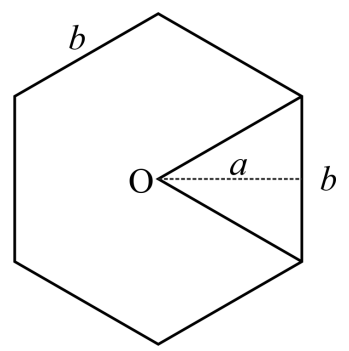

Figura 1.3: Área de un polígono regular

Uniendo el centro $O$ con los vértices se obtiene $n$ triángulos congruentes, con base $b$ y altura $a$. Luego, si $A$ es el área del polígono regular, se tiene ( $n$ veces)

$$
A=\frac{1}{2} b a+\cdots+\frac{1}{2} b a=\frac{1}{2} a(b++b)=\frac{1}{2} a P .
$$

Ahora, la idea esencial es que este proceso puede ser continuado indefinidamente; acá está la esencia del conocido método del "agotamiento" (exhaución) de Eudoxo.

1.1. Método de Exhaución de Eudoxo. En la Antigua Grecia se calculaban áreas, volúmenes y longitudes de arcos; ellos fueron, en algún sentido, los pioneros del cálculo integral. En esta dirección el método del agotamiento o "Exhaución" de Eudoxo (408 - 355 A.C.) fue vital para obtener resultados en tales cálculos.

Proposición 1.1. «Si de cualquier magnitud se substrae una parte no menor que su mitad, y de esta diferencia se substrae otra parte no menor que su mitad, y si así continuamos habrá una magnitud restante que será menor que cualquier magnitud preasignada $\gg$.

En esta dirección se tiene el

Lema 1.1. «La diferencia en área entre un círculo y un polígono regular inscrito puede ser hecho tan pequeño como se desee $\gg$.

Demostración:

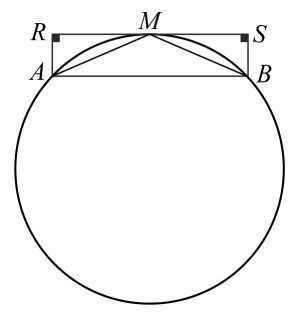

Figura 1.4: El método de la Exhaución 
Sea $\overline{A B}$ un lado de un polígono regular inscrito y $M$ el punto medio del arco $A B$. Se tiene

$$
\text { area } \triangle A M B=\frac{1}{2} \text { área } \square A B S R \text {. }
$$

Entonces se obtendrá, duplicando el número de lados del polígono, que el área del polígono se incrementará en mas de la mitad de la diferencia entre el área del círculo y el polígono.

Luego, continuando duplicando el número de lados con la frecuencia deseada, podemos hacer la diferencia en áreas del círculo y del polígono tan pequeño como se desee.

Apliquemos el lema a otro enfoque de la Proposición XII.2. que enunciamos en la forma: "Si $A_{1}$ y $A_{2}$ son las áreas de dos círculos que tienen diámetros $D_{1}$ y $D_{2}$, entonces

$$
A_{1}: A_{2}=D_{1}^{2}: D_{2}^{2} "
$$

Demostración: Supongamos que tuviéramos $A_{1}: A_{2}>D_{1}^{2}: D_{2}^{2}$. Entonces podemos inscribir en la primera circunferencia un polígono regular cuya área $P_{1}$ difiere de $A_{1}$ en una pequeña magnitud tal que $P_{1}: A_{2}>D_{1}^{2}: D_{2}^{2}$. Inscribamos en la segunda circunferencia un polígono regular, similar al anterior polígono, y sea $P_{2}$ el área del círculo asociado. Entonces, (se sabe para polígonos regulares similares) $P_{1}: P_{2}>D_{1}^{2}: D_{2}^{2}$

Entonces se tendrá $P_{1}: A_{2}>P_{1}: P_{2}$, esto es, $P_{2}>A_{2}$, lo que es absurdo. De un modo similar, $A_{1}: A_{2}<D_{1}^{2}: D_{2}^{2}$ nos llevaría a una contradicción.

Conclusión: $A_{1}: A_{2}=D_{1}^{2}: D_{2}^{2}$. De este modo, en general, $A=k D^{2}$, donde $k=\frac{1}{4} \pi$.

Área del Círculo. $A=\pi r^{2}$.

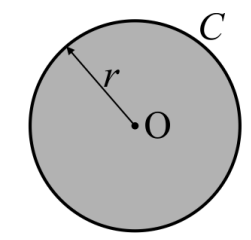

$A=$ área del círculo

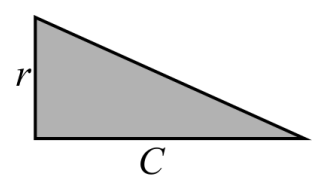

$T=$ área del triángulo

Figura 1.5: Arquímedes: área del círculo

Proposición 1.2. El área de cualquier círculo es igual al área del triángulo rectángulo en el cual uno de los lados que forma el ángulo recto es igual al radio y el otro es la circunferencia, asociada al círculo.

Demostración: Arquímedes comienza con un círculo con centro $O$, radio $r$ y circunferencia $C$; y con un triángulo rectángulo teniendo como base la longitud de $C$ y altura de longitud $r$.

El objetivo es probar que $A=T$.

En efecto: Arquímedes ya conocía que $T=\frac{1}{2} r C$ y por reducción al absurdo supone que $A>T$.

Entonces, $A-T$ es alguna cantidad positiva; Arquímedes conocía que inscribiendo un cuadrado en su círculo y repitiendo vía bisección de sus lados, él podía llegar a un polígono regular inscrito en el círculo cuya área difiere del área del círculo es una magnitud menor que el número positivo $A-T$. Esto es, $A$-área del polígono inscrito $<A-T$. Luego, $T<$ área del polígono inscrito.

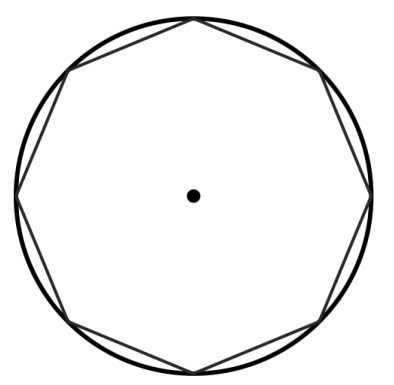

Figura 1.6: Hacia el área del círculo

Ahora se observa (ver figura 1.6) que el perímetro $P$ del polígono es menor que $C$, y que $a<r$ ( $a$ apotema). Luego, área del polígono inscrito $=\frac{1}{2} a P<\frac{1}{2} r C=T$.

De esta manera Arquímedes llega a una contradicción pues arribó a: $T<$ área del polígono inscrito y $T>$ área del polígono inscrito. 
De esta manera $A>T$ no es posible.

Supongamos ahora que $A<T$.

De esta manera, Arquímedes asume que podemos considerar $T-A$ como el exceso del área del triángulo sobre la del círculo. Por otro lado, se sabe que se puede circunscribir un polígono regular tal que el área del "polígono menos círculo" sea menor que $T-A$.

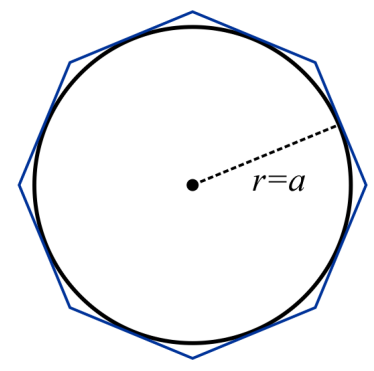

Figura 1.7: Hacia el área del Círculo

De esta manera, área del polígono circunscrito $-A<T-A$.

Luego, área del polígono circunscrito $<T$. Pero $r=a$ y $P>C$; entonces, área del polígono circunscrito $=\frac{1}{2} a P>\frac{1}{2} r C=T$.

De esta manera se llega a una contradicción. Conclusión. $A=T$.

Observación 1.2. $A=\frac{1}{2} r C=\frac{1}{2} r 2 \pi r=\pi r^{2}$.

Por razones didácticas veamos el caso de un polígono de 16 lados inscrito en una circunferencia de radio 1. ¿Cuánto valdrá $\pi$ en este caso?

\section{Solución.}

Usando reiteradamente el teorema de Pitágoras, la figura nos lleva a $A C=\sqrt{2-\sqrt{2}}$.

Por tanto, si $n=8$ tendremos

Esto es, $\pi \simeq 3,061467$

$$
\pi=\frac{C}{D} \simeq \frac{\text { perimetro }}{D}=\frac{8 \sqrt{2-\sqrt{2}}}{2}=4 \sqrt{2-\sqrt{2}}
$$

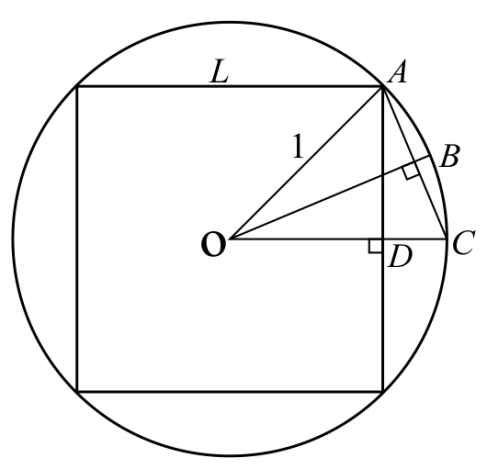

Figura 1.8: Hacia el valor de $\pi$

Veamos el caso $n=16$. Ahora el lado del polígono es $\overline{A B}$. Nuevamente por Pitágoras se tiene que

Entonces.

$$
A B=\sqrt{2-\sqrt{4-A C^{2}}}=\sqrt{2-\sqrt{2+\sqrt{2}}} .
$$

$$
\pi \simeq \frac{\text { perimetro }}{D}=\frac{16 \sqrt{2-\sqrt{2+\sqrt{2}}}}{2} \simeq 3,121445 \ldots
$$

Para $n=36$ se calcula $\pi \simeq 3,136548 \ldots$, y para $n=64$ se tiene $\pi \simeq 3,140331 \ldots$

Ejemplo 1.2. Arquímedes. Sobre la esfera y el cilindro.

Arquímedes encontró una hermosa relación entre el volumen del cilindro con la de la esfera inscrita en él. Su tratado sobre la esfera y el cilindro fue escrito en dos libros. Usa el método de exhaución en el cálculo de ciertas áreas y volúmenes. La idea es inscribir y circunscribir figuras que delimiten a la figura curvilínea que ha de ser medida. Veamos ahora como Arquímedes encuentra la superficie de un cono recto 
(Proposición 14).

Sea A la base del cono, $C$ una línea recta igual a su radio; $D$ es una línea igual al generador del cono, y $E$ es la media proporcional de $C$ y D. B es un círculo con radio $E$.

Arquímedes prueba que: «la superficie del cono es igual a la del círculo cuyo radio es la media proporcional entre el generador del cono y el radio de la base $\gg$.

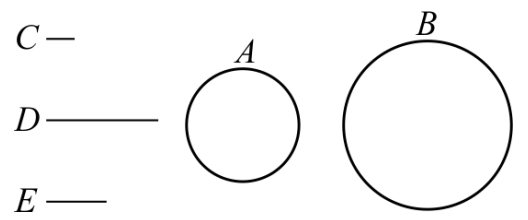

Figura 1.9: Arquímedes, superficie del cono

Si $S$ es la superficie del cono, entonces se debe probar que $S=B$. (Remarcamos que B expresa, también, el área del círculo de radio $E$ ).

El argumento usado por Arquímedes fue: supongamos que $S \neq B$. Entonces discute los casos $B<S y$ $S<B$.

Caso $B<S$, Arquímedes circunscribe un polígono regular a $B$ e inscribe un polígono similar en $B$ tal que la razón del primer polígono al segundo es menor que $\frac{S}{B}$. Este resultado es su Proposición 5. Ahora describe alrededor de A un polígono similar y construye a partir de esto una pirámide que circunscribe al cono. Entonces se tiene

Luego,

$$
\frac{\text { polígono alrededor de } A}{\text { polígono alrededor de } B}=\frac{C^{2}}{B^{2}}=\frac{C}{D}=\frac{\text { polígono alrededor de } A}{\text { superficie de la pirámide }} .
$$

Superficie de la pirámide = polígono alrededor de B.

Por otro lado sabemos que $\frac{\text { polígono alrededor de } A}{\text { polígono en } B}<\frac{S}{B}$.

Luego,

$$
\frac{\text { superficie de la pirámide }}{\text { polígono en } B}<\frac{S}{B} \text {. }
$$

Pero esto no es posible ya que

superficie de la pirámide $>S$ y polígono en $B<B$.

Conclusión: $B$ no es menor que $S$.

Caso $B>S$. Nuevamente, Arquímedes circunscribe e inscribe polígonos regulares similares en $B$ tal que la razón del primer al segundo es menor que $\frac{B}{S}$. Luego inscribe en $A$ un polígono similar y construye sobre A una pirámide inscrita. Entonces se tiene, $\frac{\text { polígono en } A}{\text { polígono en } B}=\frac{C^{2}}{E^{2}}=\frac{C}{D}>\frac{\text { polígono en } A}{\text { superficie de la pirámide }}$,

(la desigualdad es debido a que la razón $\frac{C}{D}$ es mayor que la razón de las perpendiculares desde el centro de A y desde el vértice de la pirámide, respectivamente, sobre cualquier lado del polígono en A).

Por lo tanto,

Superficie de la pirámide > polígono en $B$.

Pero, se tiene también $\quad \frac{\text { polígono alrededor de } B}{\text { poligono en } B}<\frac{B}{S}$,

y por lo tanto, “a fortiori”, $\frac{\text { polígono alrededor de } B}{\text { superficie de la piramide }}<\frac{B}{S}$.

Esto no es posible ya que:

polígono alrededor de $B>B$ y superficie de la pirámide $<S$.

Por lo tanto, $B$ no es mayor que $S$.

Conclusión final: $S=B$.

Observación 1.3. Remarcamos que cuando se dice: "la superficie” se debe entender por "el área de la superficie”. 
Como hemos manifestado, Arquímedes trabajó con la geometría del espacio, con sólidos geométricos en el espacio tridimensional, obteniendo resultados maravillosos con tales sólidos, en particular con la esfera y el cilindro.

1.1.1. Área de la Superficie Lateral de un Cilindro (recto).. Dado un cilindro $H$, lo cortamos verticalmente y lo extendemos sobre un plano. Al hacer esto se obtiene, un rectángulo con lados $2 \pi r$ y $h$.

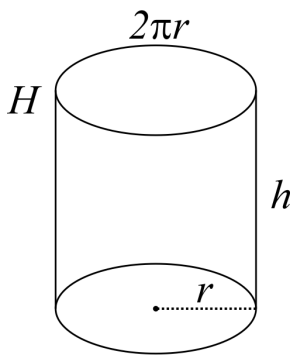

Figura 1.10: Superficie lateral del cilindro

Luego, área de la superficie lateral de $H=(2 \pi r) h=2 \pi r h$.

1.1.2. Área de la Superficie Lateral de un Cono (recto).. Sea $K$ un cono cuyo círculo base tiene radio $r$, y sea $g$ su generatriz. Esta vez se corta al cono desde un punto de la circunferencia del círculo base hacia el vértice. Desenrollamos la figura y la aplanamos, obteniéndose un sector circular como se muestra en la figura (ii).

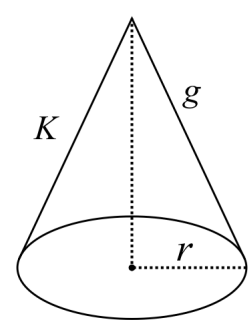

(i)

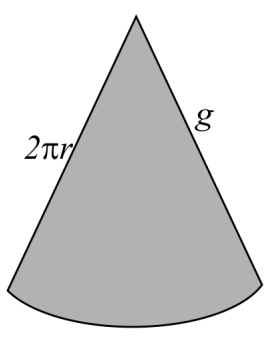

(ii)

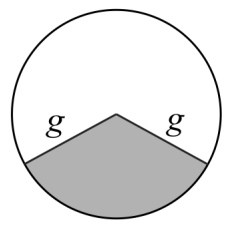

(iii)

Figura 1.11: Superficie lateral del cono

La idea ahora es considerar este sector como una parte del círculo de radio $g$. Arquímedes conocía la siguiente relación:

$$
\frac{\text { área del sector }}{\text { area del círculo }}=\frac{\text { longitud del arco del sector }}{\text { circunferencia del círculo }} \text {. }
$$

Observemos que el área del círculo es $\pi g^{2}$, y su circunferencia tiene longitud $2 \pi g$; además, la longitud del arco del sector es igual a $2 \pi r$. Luego, de tal relación se obtiene,

$$
\frac{\text { area del sector }}{\pi g^{2}}=\frac{2 \pi r}{2 \pi g}=\frac{r}{g}
$$

Por lo tanto, área del sector $=\frac{r}{g}\left(\pi g^{2}\right)=\pi r g$.

Conclusión: área de la superficie lateral del cono $=\pi r g$.

1.1.3. Área de la Superficie Lateral del Tronco de Cono.. En su estudio de la esfera, Arquímedes necesitó conocer a la superficie lateral del tronco de cono (recto). Como sabemos un tronco de cono se obtiene cuando un cono es cortado por un plano paralelo a la base y se retira la parte superior (un cono más pequeño). 

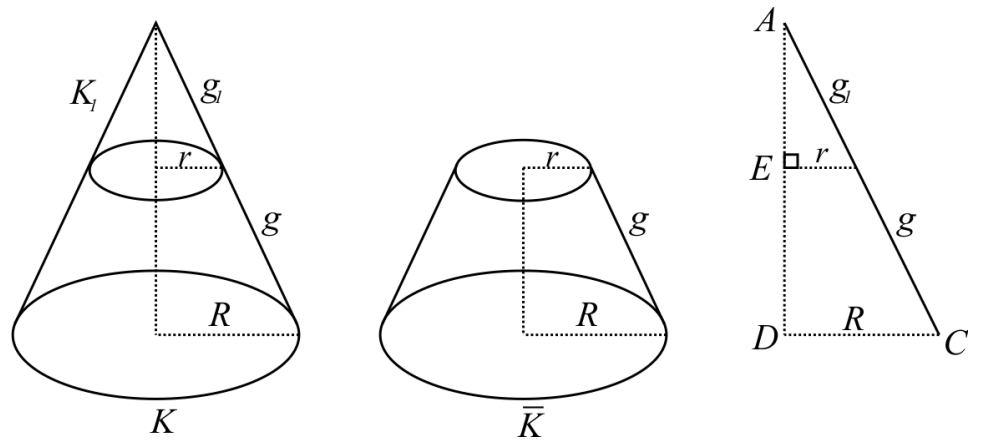

Figura 1.12: Superficie lateral del tronco de cono

Sea el tronco de cono $\bar{K}$, el que proviene del cono $K$, cuyos elementos se muestran en las figuras adjuntas. Tenemos,

área de la superficie lateral del tronco de cono $=$

(área superficie lateral del cono $K)$ - (área superficie lateral del cono $\left.K_{1}\right)$

$=\pi R\left(g+g_{1}\right)-\pi r g_{1}=\pi\left(R g+\left(R g_{1}-r g_{1}\right)\right)$.

Ahora observemos a los triángulos rectángulos semejantes $A E F$ y $A D C$.

Se tiene, $\frac{g_{1}}{g+g_{1}}=\frac{r}{g}$, es decir, $R g_{1}-r g_{1}=r g$. Por tanto,

área de la superficie lateral del tronco de cono $=\pi(R g+r g)=\pi g(R+r)$.

1.1.4. Área de la Superficie de la Esfera.. El cálculo del área de la superficie de una esfera fue un difícil reto al genio de Arquímedes. Es asombrosa la metodología que siguió para lograr su objetivo, pues en vez de considerar a la esfera tridimensional comienza analizando a una "tajada" infinitesimal, es decir, estudia al círculo bidimensional. Miremos con atención la figura A.l adjunta:

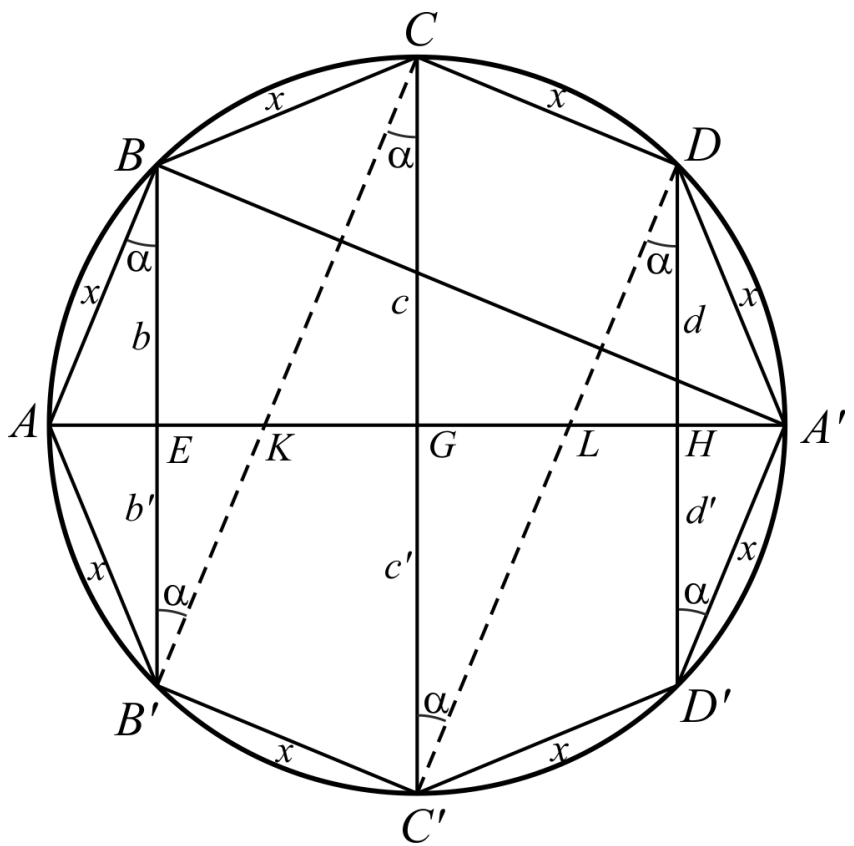

Figura 1.13: Arquímedes, superficie de la esfera.

Se considera un círculo de radio $r$ y diámetro $A A^{\prime}$, en donde se inscribe un polígono regular con un número par de lados (en el caso de la figura consideramos a un octógono regular); cada lado mide $x$. El siguiente argumento es válido para cualquier número par de lados. Entonces sea el octógono $A B C D A^{\prime} D^{\prime} C^{\prime} B^{\prime}$.

Arquímedes traza las líneas verticales $B B^{\prime} ; C C^{\prime}$ y $D D^{\prime}$ las que cortan al diámetro $A A^{\prime}$ en los puntos $F, G$ y $H$ respectivamente. Además considera las líneas punteadas $B^{\prime} C$ y $C^{\prime} D$ que cortan a tal diámetro en $K$ y $L$. Además traza la línea $A^{\prime} B$ (¿para qué?). De esta manera se obtiene un panorama algo complicado de 
muchas figuras geométricas. ¿Qué hacer ahora?, ¿qué hizo Arquímedes? ...

¿Cuál será el papel de la línea $A^{\prime} B$, cuya longitud es $y$ ?

Nota. En primer lugar observó que $B F=F B^{\prime}, C G=G C^{\prime}$ y $D H=H D^{\prime}$, que llamó $b, c$ y $d$ a las longitudes respectivas $(B F=b, \ldots)$. Ahora él apela a un resultado que está en el Libro III de los Elementos de Euclides: "los ángulos en los círculos que intersectan arcos iguales, son iguales entre si".

Luego, desde que el polígono es regular, se tendrá

$$
\angle B A^{\prime} A=\angle A B B^{\prime}=\angle B B^{\prime} C=\ldots
$$

ángulos que se denotan $\operatorname{con} \alpha$.

Por otro lado, Arquímedes observa que los triángulos $\triangle A B A^{\prime}$ y $\triangle A F B$ son semejantes (ambos comparten el $\measuredangle B A A^{\prime}$ y ambos tienen un ángulo que mide $\alpha$ ).

Luego, por Tales, se tiene:

Por lo tanto,

$$
\frac{A F}{B F}=\frac{A B}{A^{\prime} B} \quad \text { ó } \quad \frac{A F}{b}=\frac{\chi}{y}
$$

$$
x b=y(A F) \quad \text { (i) }
$$

Similarmente, el $\triangle A F B$ es semejante al $\triangle K F B^{\prime}$ (ambos contienen un ángulo que vale $\alpha, \mathrm{y} \measuredangle A F B=$ $\left.\measuredangle K F B^{\prime}\right)$. Entonces,

Luego,

$$
\frac{F K}{B^{\prime} F}=\frac{A F}{B F} \quad \text { ó } \quad \frac{F K}{b}=\frac{A F}{b}=\frac{x}{y} .
$$

$$
x b=y(F K)
$$

Continuando se observa que el $\Delta K F B^{\prime}$ es semejante al $\Delta K G C$ (¿porqué? ...)

Luego,

$$
\frac{K G}{C G}=\frac{F K}{B^{\prime} F} \quad \text { ó } \quad \frac{K G}{c}=\frac{F K}{b}=\frac{x}{y}
$$

Continuando con el resto de triángulos semejantes, se obtienen las siguientes relaciones:

$$
\begin{aligned}
& x c=y(G L) \quad \text { (iv) } \\
& x d=y(L H) \quad \text { (v) } \\
& x d=y\left(H A^{\prime}\right) \quad \text { (vi) }
\end{aligned}
$$

Ahora Arquímedes suma: (i) + (ii) $+\ldots+$ (vi) para obtener

$x b+x b+x c+x c+x d+x d=y\left(A F+F K+K G+G L+L H+H A^{\prime}\right)$,

esto es,

$x(2 b+2 c+2 d)=y\left(A A^{\prime}\right)=2 r y$.

Y ahora, ¿cuál será la estrategia de Arquímedes ?, ¿cómo usar (+)? El gran geómetra siracusano considera una esfera, la que es obtenida haciendo girar (gran idea!) la figura anterior alrededor del eje horizontal $A A^{\prime}$. Ya Euclides decía que en este caso se obtiene una esfera y el polígono de revolución genera un sólido que consiste en una serie de troncos de conos (cuyas áreas de sus superficies laterales ya conocía Arquímedes) y con dos conos pegados en los extremos. Ver Figura A.2. Se observa que la generatriz de cada cono y de cada tronco de cono es $x$. Ahora estamos en condiciones de calcular el área de la superficie de este sólido. Bien, veamos al cono de la izquierda: $b$ es el radio de la base, $x$ es la generatriz, luego el área de la superficie (lateral) de ese cono es $\pi x b$. Continuando, el tronco de cono de la izquierda tiene generatriz $x, b$ como radio superior, $c$ como radio inferior; luego, el área de su superficie (lateral) es $\pi x(b+c)$. El tronco de cono de la derecha es tal que el área de su superficie (lateral) es igual a $\pi x(c+d)$, y

el cono de la derecha tiene una superficie de área (lateral) igual a $\pi x d$.

\section{Conclusión:}

área de la superficie del sólido inscrito $=$

$$
\pi x b+\pi x(b+c)+\pi x(c+d)+\pi x d=\pi x(2 b+2 c+2 d) .
$$




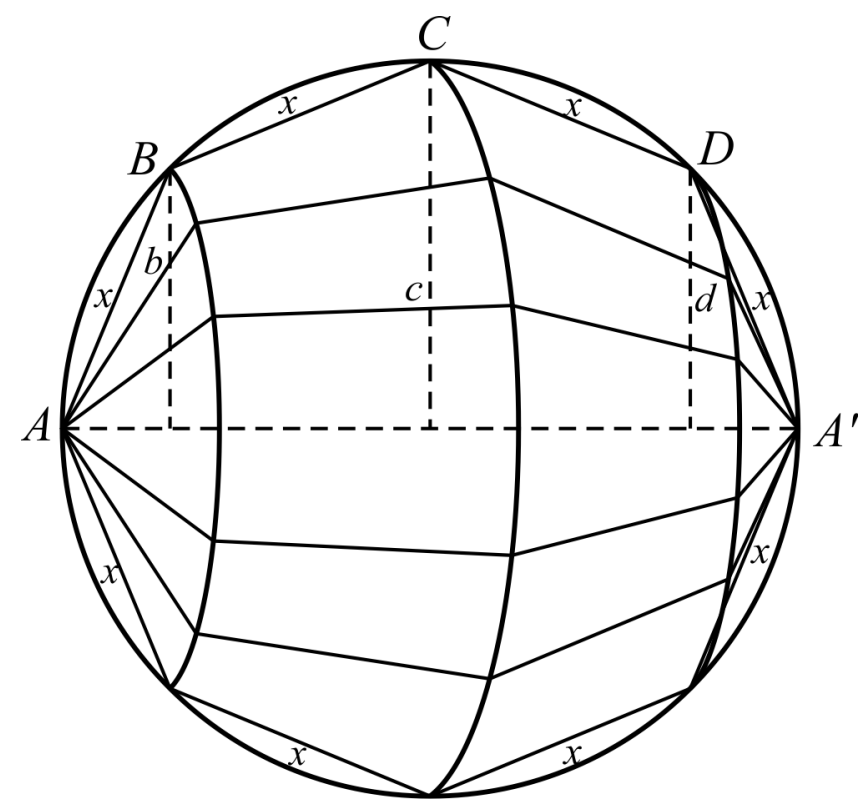

Figura 1.14: Área de la superficie de la esfera

Observemos que en esta fórmula está (+)!, y por tanto se tiene:

área de la superficie del sólido inscrito $=\pi(2 r y)$.

Observemos que en esta interesante fórmula aparece $y$, la longitud de la enigmática línea $A^{\prime} B$. También esa fórmula nos sugiere porqué Arquímedes impuso la condición de que el polígono regular tenga un número par de lados, pues así cada radio $b, c$ y $d$ es compartido por dos sólidos.

Ahora, ¿cómo obtener el área de la superficie de la esfera? Posiblemente el lector ya intuído el paso siguiente; esto ayudado por la idea de límite que ahora disponemos, pero que en el tiempo de Arquímedes no existía (al menos formalmente!). Acá entró a trabajar la gran intuición de este notable geómetra. Es natural sentir que el área de la superficie del sólido (del octógono en este caso) es una aproximación del área de la superficie de la esfera, y también, es un sentimiento confiable creer que si el número de lados (par) del polígono regular aumenta en número, entonces tal área va aproximándose al área deseada. Esto fue la etapa crucial en el argumento de Arquímedes.

Con los argumentos actuales se puede escribir:

área de la superficie de la esfera = lím (área de las superficies de los sólidos inscritos) $=\operatorname{lím} \pi(2 r y)$.

Nota. Enfatizamos que el límite es tomado cuando el número de lados del polígono crece y crece. Ahora, otra vez apelamos a los "sentimientos matemáticos": viendo la Figura A.2 se observa que

$$
\lim y=\lim A^{\prime} B=A^{\prime} A=2 r .
$$

Así, Arquímedes llega a la bella fórmula:

$$
\text { área de la superficie de la esfera }=\lim \pi(2 r y)=\pi 2 r l i m y=4 \pi r^{2} \text {. }
$$

En [4], el lector puede encontrar un amplio material sobre Arquímedes; el Prof, T. Heath fue un gran investigador sobre los trabajos del genial matemático griego. Nosotros hemos seguido, también, a [3]. Es claro que la prueba dada trata de conservar el pensamiento de Arquímedes pero como hemos visto se han usado argumentos y notaciones que en su época no existían. Arquímedes expresó su resultado diciendo: «La superficie de cualquier esfera es cuatro veces el círculo máximo de ella $\gg$, algo que ya hemos mencionado.

1.1.5. Esfera Inscrita en un Cilindro.. Arquímedes prueba el siguiente bello resultado, el gran teorema sobre la esfera y el cilindro: 
$\ll$ Cualquier cilindro que tiene su base igual al círculo máximo de la esfera y la altura igual al diámetro de la esfera es la mitad de grande más que la superficie de la esfera $\gg$.

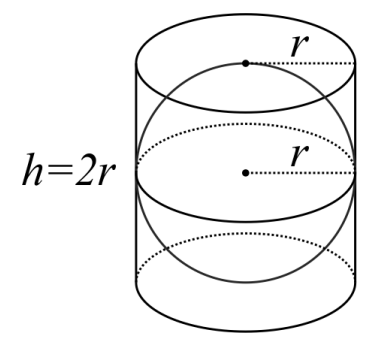

Figura 1.15: El gran teorema de Arquímedes

¿Cuál es la interpretación de "la mitad de grande más"?

Para Arquímedes esto significaba:

"superficie del cilindro = superficie de la esfera $+\frac{1}{2}$ (superficie de la esfera).

Observemos que ya se ha probado:

"área de la superficie del cilindro $=2 \pi r h "$.

Luego, en este caso en que la esfera está inscrita en el cilindro y que se tiene $h=2 r$, el área de la superficie lateral del cilindro es $2 \pi r(2 r)=4 \pi r^{2}$, que es el área de la superficie de la esfera.

En este caso se debe enfatizar que Arquímedes cuando habla del área del cilindro, él está incluyendo ahora a la parte superior y a la inferior.

De esta manera se tiene,

superficie total del cilindro $=$ superficie lateral + superficie parte superior + superficie parte inferior

$$
=4 \pi r^{2}+\pi r^{2}+\pi r^{2}=6 \pi r^{2} .
$$

Por lo tanto, la tesis del teorema de Arquímedes:

superficie total del cilindro = mitad más que la superficie de la esfera,

toma la forma: $6 \pi r^{2}=E+\frac{1}{2} E$, donde $E$ representa el área de la superficie de la esfera. Luego, $12 \pi r^{2}=$ $2 E+E=3 E$, esto es, $E=4 \pi r^{2}$, algo que sabemos es cierto. De esta manera se tiene verificado al teorema de Arquímedes.

1.1.6. ¿Qué se puede afirmar respecto a los volúmenes?. Seguimos con el caso de una esfera inscrita en un cilindro. Se sabía que el volumen de un cilindro, en general, es $\pi r^{2} h$. Asi, en tal caso se tendrá, volumen del cilindro $=\pi r^{2}(2 r)=2 \pi r^{3}$

Arquímedes conocía su

Proposition 34:it $\ll$ cualquier esfera es igual a cuatro veces el cono, el cual tiene como base al más grande círculo en la esfera y su altura es igual al radio de la esfera $\gg$.

Arquímedes conocía también que: "Volumen del cono $=\frac{1}{3} \pi r^{2} h$ ", y en el caso de la Proposición 34 se tiene: "Volumen del cono $=\frac{1}{3} \pi r^{3}$ ".

Por tanto la proposición 34 nos dice:

$$
\text { Volumen de la esfera }=4(\text { volumen del cono })=\frac{4}{3} \pi r^{3} .
$$

Regresando a la formula (+) se tiene,

$$
\text { Volumen del cilindro }=2 \pi r^{3}=\frac{3}{2}\left(\frac{4}{3} \pi r^{3}\right) .
$$

Conclusión: $\ll$ Volumen del cilindro $=\frac{3}{2}$ (Volumen de la esfera) $\gg$.

Es decir, nuevamente: "Volumen del cilindro = volumen de la esfera $+\frac{1}{2}$ (volumen de la esfera)". En otras palabras, el volumen del cilindro es la "mitad de grande más" que el volumen de la esfera. Un bello resul- 
tado.

2. Siglo XVII. El Cálculo Infinitesimal. En [6] hemos visto diversos ejemplos históricos sobre la evolución de ideas y métodos que condujeron a la creación del cálculo infinitesimal por Leibniz y por Newton. En particular, Newton hizo de las series infinitas su recurso para desarrollar el cálculo; Leibniz también usó las series en sus argumentos, que como observamos se estaba trabajando con el "infinito". Leibniz nos dice ([1], pag. 31):

"He observado -escribe Leibniz a Fontenelle el 12 de julio de 1702-, que hay

dos maneras de llegar a las sumas de las áreas o a las rectificaciones de curvas por el infinito: una por los infinitésimos, o cantidades elementales, de las que se busca su suma; la otra por una progresión de términos ordinarios de los que se busca la suma o bien su terminación cuando, por último, termina en lo que envuelve al infinito..., y este método difiere (toto genere) de nuestro cálculo de las diferencias, de las sumas".

De esta manera se podría decir que la investigación de las series infinitas fue fundamental en la creación del cálculo infinitesimal. Con el manejo de las series los matemáticos sentían que podían manejar el infinito pero estaba faltando un concepto general de función! La creación del cálculo fue un hecho feliz para la humanidad por todas las consecuencias que sabemos. Además, nace el análisis matemático. Veamos un panorama post-Newton.

En el siglo XVII matemáticos notables introdujeron nuevas ideas y teorías fundamentales como son la geometría analítica y el cálculo infinitesimal, así como el álgebra, la que fue un argumento superior en la solución de problemas geométricos. La matemática se la investiga intrínsecamente en relación con el mundo físico, así el cálculo se aplica con éxito en la física-matemática, en la astronomía, en la mecánica. Las sociedades matemáticas y las revistas matemáticas ayudaron mucho en la comunicación de los científicos. La mecánica celeste se desarrolla aceleradamente gracias a los trabajos de Newton. La intuición y la visión física guiaron diversas investigaciones matemáticas. Aun no existía el rigor analítico; hubo cierta confusión de notaciones, conceptos y en el proceso creativo. El siglo XVIII se caracteriza por el desarrollo de la mecánica y el cálculo diferencial e integral. Así mismo, de descubrir nuevas aplicaciones vía las ecuaciones diferenciales, ordinarias y parciales; se estudian las series infinitas, la geometría diferencial y el cálculo de variaciones. Por el lado del álgebra se estudian sistemáticamente las ecuaciones algebraicas de grado superior a cuatro; la teoría de números y los números complejos son investigados.

Una notable familia, la familia Bernoulli, contribuyó mucho en el progreso de la matemática a través de trece de sus miembros. Nicolaus fue el primero y nació en 1623; Jean Gustave fue el último y nació en 1811, es decir, tuvieron vivencia durante tres siglos. Los que más destacaron fueron, Jacques I. (1654-1705), su hermano Jean I. (1667-1748) y un hijo de Jean I., Daniel I. (1700-1782). A Jacques le debemos la palabra "integral", nombre que sugirió a Leibniz; hizo contribuciones en las series infinitas. A los hermanos Jacques y Jean, además de sus propias contribuciones, les debemos la difusión de la obra de Leibniz. Jean tuvo un excepcional alumno: Leonardo Euler, de quien nos ocuparemos más tarde. En Francia, el marqués de L'Hospital (1661-1704) escribió dos breves textos sobre cálculo diferencial e integral y es conocido por la llamada "regla de L-Hospital", la que fue descubierta por Jean I. El caso de Daniel I. es anecdótico pues su padre, Jean I., quiso que el hijo estudiara comercio pero este se reveló y estudió matemática.

Daniel I. fue un gran filósofo natural, trabajó en física; en sus estudios sobre elasticidad utilizó ecuaciones diferenciales, tanto ordinarias como parciales. Contribuyó en la teoría de probabilidades e hizo aplicaciones en la economía; contribuyó también en el cálculo de las funciones trigonométricas. Algo notable que Daniel hizo fue formular el problema de la cuerda vibrante usando ecuaciones en derivadas parciales; este problema podría ser considerado como la fuente del futuro análisis de Fourier. El desarrollo de la matemática, y del cálculo, se deben también a otros matemáticos del siglo XVIII. Citemos algunos.

2.0.1. Abraham De Moivre - [(1667-1754)] fue amigo de Newton y lo recordamos por su familiar fórmula $\left(\cos \theta \pm i \operatorname{sen} \theta^{n}=\cos n \theta \pm i \operatorname{sen} \theta\right.$.

2.0.2. Roger Cotes. [(1682-1716)] vivió solo hasta los 34 años pero nos legó trabajos importantes y muchas ideas por elaborarse. La matemática perdió a una promesa matemática; Newton le tuvo un gran aprecio; dijo de él: "si viviera Cotes podríamos haber sabido algo". Cotes trabajó la integración de fracciones racionales vía descomposición en facciones parciales; dedicó gran parte de su tiempo en la preparación de la segunda edición de los Principia de Newton.

2.0.3. Brook Taylor. [(1685-1731)] quien es conocido actualmente por la "serie de Taylor" (1715); Taylor admiró el genio de Newton. En 1719, abandona sus estudios matemáticos; el joven Maclaurin los ha 
de continuar.

El siglo XVIII ha de ser el siglo del gran matemático Leonardo Euler.

Ejemplo 2.1. Las Series Infinitas.

(i) Las series infinitas aparecieron en algunos trabajos de los antiguos griegos, así; • en una paradoja de Zenón aparece la serie infinita

$\frac{1}{2}+\frac{1}{2^{2}}+\frac{1}{2^{3}}+\frac{1}{2^{4}}+\frac{1}{2^{5}}+\ldots=1$

Arquímedes se enfrentó a la serie $1+\frac{1}{4}+\frac{1}{4^{2}}+\frac{1}{4^{3}}+\frac{1}{4^{4}}+\ldots$ en su trabajo para hallar el área de un segmento parabólico; obtuvo que la suma "infinita" es igual a $\frac{4}{3}$.

(ii) Edad Media. • Richard Suiseth (1350) probó que $\frac{1}{2}+\frac{2}{2^{2}}+\frac{3}{2^{3}}+\frac{4}{2^{4}}+\ldots=2$.

Nicole Oresme (1350) descubre la divergencia de la serie armónica $1+\frac{1}{2}+\frac{1}{3}+\frac{1}{4}+\frac{1}{5}+\ldots$

Matemáticos indios encontraron la serie $x-\frac{x^{3}}{3}+\frac{x^{5}}{5}-\frac{x^{7}}{7}+\ldots=t g^{-1} x$.

En particular se obtiene una aproximación del valor de $\pi$ :

$\frac{\pi}{4}=1-\frac{1}{3}+\frac{1}{5}-\frac{1}{7}+\frac{1}{9}-\ldots$

(iii) Mercator (1668) inicia la teoría de series de potencias con la publicación de la serie

$\log (1+x)=x-\frac{x^{2}}{2}+\frac{x^{3}}{3}-\frac{x^{4}}{4}+\frac{x^{5}}{5}-\ldots$

la cual fue obtenida integrando la serie (geométrica)

$$
1-x+x^{2}-x^{3}+x^{4}-x^{5}+\ldots=\frac{1}{1+x} .
$$

Así, en esta dirección el paso fundamental lo dio Nicolás Mercator (1620-1687) cuando publica su célebre obra sobre los logaritmos "Logarithmo-Technia".

En (iii), Mercator estudia la cuadratura de la hipérbola equilátera, obteniendo tal serie vía una división de 1 por $1+x$, y así se pasa (de alguna manera) de la "aritmética del infinito" al "álgebra del infinito". Por otro lado, en general ¿esas infinitas sumas "convergen" a algo? En esa época aún no había casi preocupación por esta cuestión, y por ello habrían de surgir ciertas "rarezas".

Resaltamos que la contribución de Mercator condujo a usar el álgebra en las cuestiones del análisis infinitesimal! Recordemos que por aquella época Newton también trabajaba con series de un modo similar; obtuvo, por ejemplo,

$$
\left(1-x^{2}\right)^{1 / 2}=1-\frac{1}{2} x^{2}-\frac{1}{8} x^{4}-\frac{1}{16} x^{6}-\ldots
$$

y tuvo la paciencia de multiplicar la serie con ella misma y obtuvo $1-x^{2}$.

(Ver [9] para mayores detalles).

3. Siglo XVIII. Consolidación del Cálculo. Euler. En el siglo XVIII, y primeros años del XIX, el cálculo infinitesimal habría de completarse pero sin clarificar aun algunas nociones básicas como, por ejemplo, el concepto de "función", del cual se tenía una idea bastante vaga. Tampoco estaban precisas las nociones de límite, continuidad, infinito, ... Pero, si el siglo XVII tuvo a Newton, el XVIII tuvo al extraordinario matemático Leonardo Euler (1707-1783), quien fue la figura central del periodo algorítmico, como fue su época, periodo en que el análisis infinitesimal y el análisis algebraico se independizan. La matemática va formalizándose, aun no del todo riguroso. Euler fue el gran matemático que hizo del siglo XVIII la antesala de un nuevo siglo, el XIX.

En el siglo XVIII los matemáticos estuvieron preocupados y presionados por los avances de la física y de la astronomía; ciertas investigaciones condujeron a series y a ecuaciones diferenciales (ordinarias y parciales); también aparecieron algunas representaciones en series trigonométricas y esto fue el inicio de nuevas conquistas, sobre todo en las aplicaciones. Además, en Francia se ha de producir una etapa de gran esplendor en el transito del siglo XVIII al siguiente. Por esta época surge el análisis de Fourier. Mencionamos a algunos matemáticos de este gran periodo:

- D'Alembert (1717-1783), quien está relacionado al problema de la cuerda vibrante; este problema originó una polémica sobre la naturaleza de las funciones consideradas.

- Alexis C. Clairaut (1713-1765) hizo importantes aportes en el futuro análisis armónico. 
Afirma que: "toda función puede escribirse en la forma $f(x)=A_{o}+2 \sum_{n=1}^{\infty} A_{n} \cos n x$, donde $A_{n}=\frac{1}{2 \pi} \int_{o}^{2 \pi} f(x) \cos n x d x "$.

- Joseph-Louis Lagrange (1736-1813). En 1788 Lagrange publica su obra: "Mecánica Analítica”, escrita con un lenguaje estrictamente matemático, en donde aplica el reciente análisis matemático a la mecánica de los cuerpos sólidos.

- Pierre Simón Laplace (1749-1827) quien fue famoso por su trabajo en astronomía; escribió su excelente "Mecánica Celeste" (5 vols), razón por lo cual se le llamó "el Newton de Francia”. Con Laplace se inicia la etapa moderna del cálculo de probabilidades.

- Adrien-Marie Legendre (1752-1833), quien contribuyó en la teoría de números, en las integrales elípticas, las ecuaciones diferenciales, ... Escribió el tratado "Elementos de Geometría”.

$Y$ así tenemos muchos otros notables matemáticos.

Resumen. La matemática, en particular el análisis, tuvo un gran desarrollo durante los siglos XVII y XVIII debido a grandes matemáticos quienes fundaron la ciencia y la tecnología moderna. Sin embargo, a fines del siglo XVIII los matemáticos estuvieron descontentos con ciertas paradojas y vacíos que existían en las ideas de función, límite, continuidad, ..., el infinito.

En el siglo XIX tendremos otro “milagro griego".

\section{Ejemplo 3.1 (Series Trigonométricas).}

Euler considera la expansión

$$
\frac{1}{1-x}=1+x+x^{2}+x^{3}+\ldots
$$

y reeemplaza $x$ por el complejo $\mathrm{z}=a(\cos \phi+i \operatorname{sen} \phi)$ y obtiene la expansión

$$
\frac{1}{1-a(\cos \phi+i \operatorname{sen} \phi)}=1+[a(\cos \phi+i \operatorname{sen} \phi)]+[a(\cos \phi+i \operatorname{sen} \phi)]^{2}+[a(\cos \phi+i \operatorname{sen} \phi)]^{3}+\ldots
$$

Euler trabaja con el lado izquierdo; así, si $a=-1$ y racionalizando,

$$
\begin{aligned}
\frac{1}{1+(\cos \phi+i \operatorname{sen} \phi)} & =\frac{1}{1+(\cos \phi+i \operatorname{sen} \phi)} \frac{1+\cos \phi-i \operatorname{sen} \phi}{1+\cos \phi-i \operatorname{sen} \phi} \\
& =\frac{1+\cos \phi-i \operatorname{sen} \phi}{(1+\cos \phi)^{2}+\operatorname{sen}^{2} \phi}=\frac{1+\cos \phi-i \operatorname{sen} \phi}{2(1+\cos \phi)}=\frac{1}{2}-\frac{i \operatorname{sen} \phi}{2(1+\cos \phi)}
\end{aligned}
$$

Ahora miremos al lado derecho de (*) y recordemos al teorema De Moivre, $(\cos \phi+i \operatorname{sen} \phi)^{n}=\operatorname{cosn} \phi+$ isenn $\phi$, el que permite escribir,

$$
\begin{aligned}
1-(\cos \phi+i \operatorname{sen} \phi)+ & (\cos 2 \phi+i \operatorname{sen} 2 \phi)-(\cos 3 \phi+i \operatorname{sen} 3 \phi)+\ldots \\
& =(1-\cos \phi+\cos 2 \phi-\cos 3 \phi+\ldots)-i(\operatorname{sen} \phi-\operatorname{sen} 2 \phi+\operatorname{sen} 3 \phi-\ldots)
\end{aligned}
$$

Ahora, lo natural es igualar las partes real e imaginaria para obtener:

$\frac{1}{2}=1-\cos \phi+\cos 2 \phi-\cos 3 \phi+\ldots$ de donde se obtiene $\cos \phi-\cos 2 \phi+\cos 3 \phi-\ldots=\frac{1}{2}$.

Ahora la idea es integrar término a término esta igualdad para obtener

$$
\operatorname{sen} \phi-\frac{1}{2} \operatorname{sen} 2 \phi+\frac{1}{3} \operatorname{sen} 3 \phi-\frac{1}{4} \operatorname{sen} 4 \phi+\frac{1}{5} \operatorname{sen} 5 \phi-\ldots=\frac{1}{2} \phi+C_{1} \text {. (i) }
$$

Si $\phi=0$ se obtiene $C_{1}=0$.

Integrando esta igualdad se obtiene,

$$
\cos \phi-\frac{1}{2^{2}} \cos 2 \phi+\frac{1}{3^{2}} \cos 3 \phi-\frac{1}{4^{2}} \operatorname{sen} 4 \phi+\frac{1}{5^{2}} \operatorname{sen} 5 \phi-\ldots=-\frac{\phi^{2}}{4}+C_{2} \cdot(+)
$$

Si $\phi=0, \cos 0=1$ y entonces $C_{2}=1-\frac{1}{2^{2}}+\frac{1}{3^{2}}-\frac{1}{4^{2}}+\frac{1}{5^{2}}-\ldots$ (**).

Pero, Euler sabia que el lado derecho de (**) es igual a $\frac{\pi^{2}}{12}$.

Verifiquemos: que, en efecto, $\frac{\pi^{2}}{12}=1-\frac{1}{2^{2}}+\frac{1}{3^{2}}-\frac{1}{4^{2}}+\frac{1}{5^{2}}-\ldots .(\bullet)$

Miremos $(* *)$; sabemos que $\cos (2 \pi+\alpha)=\cos \alpha$ y poniendo $\phi=\frac{\pi}{2}$ en $(+)$ se obtiene

$$
-\frac{\pi^{2}}{16}+C_{2}=\cos \frac{\pi}{2}-\frac{1}{2^{2}} \cos \pi+\frac{1}{3^{2}} \cos \frac{3 \pi}{2}-\frac{1}{4^{2}} \cos 2 \pi+\frac{1}{5^{2}} \cos \frac{\pi}{2}-\ldots
$$


de donde $-\frac{\pi^{2}}{16}+C_{2}=\frac{1}{4}\left(1-\frac{1}{2^{2}}+\frac{1}{3^{2}}-\frac{1}{4^{2}}+\ldots\right)=\frac{1}{4} C_{2}$.

De esta igualdad se tiene que $C_{2}=\frac{\pi^{2}}{12}$, que es la igualdad $(\bullet)$

Remarquemos que los argumentos dados han sido con el objetivo de probar la descomposición (•)

Mirando $(\bullet)$ es natural preguntarse, ¿a qué es igual $1+\frac{1}{2^{2}}+\frac{1}{3^{2}}+\frac{1}{4^{2}}+\ldots$ ?

Euler resolvió esta cuestión (ver [8], pag.53).

Ejemplo 3.2 ((Euler)). Verificar que $1+\frac{1}{2^{2}}+\frac{1}{3^{2}}+\frac{1}{4^{2}}+\frac{1}{5^{2}}+\ldots=\frac{\pi^{2}}{6}$.

Prueba.

$\operatorname{Por}(\bullet)$,

$$
\begin{aligned}
\frac{\pi^{2}}{12} & =1-\frac{1}{2^{2}}+\frac{1}{3^{2}}-\frac{1}{4^{2}}+\frac{1}{5^{2}}-\frac{1}{6^{2}}+\frac{1}{7^{2}}-\frac{1}{8^{2}}+\ldots \\
& =(\text { reordenando de modo que surja la suma infinita de la tesis }) \\
& =\left(1+\frac{1}{2^{2}}+\frac{1}{3^{2}}+\frac{1}{4^{2}}+\frac{1}{5^{2}}+\frac{1}{6^{2}}+\frac{1}{7^{2}}+\frac{1}{8^{2}}+\ldots\right)-2\left(\frac{1}{2^{2}}+\frac{1}{4^{2}}+\frac{1}{6^{2}}+\frac{1}{8^{2}}+\ldots\right) \\
& =\left(\text { factorizando } \frac{1}{2^{2}} \text { en la segunda suma }\right) \\
& =\left(1+\frac{1}{2^{2}}+\frac{1}{3^{2}}+\frac{1}{4^{2}}+\frac{1}{5^{2}}+\frac{1}{6^{2}}+\frac{1}{7^{2}}+\frac{1}{8^{2}}+\ldots\right)-\frac{2}{2^{2}}\left(1+\frac{1}{2^{2}}+\frac{1}{3^{2}}+\frac{1}{4^{2}}+\ldots\right) \\
& =\frac{1}{2}\left(1+\frac{1}{2^{2}}+\frac{1}{3^{2}}+\frac{1}{4^{2}}+\frac{1}{5^{2}}+\frac{1}{6^{2}}+\frac{1}{7^{2}}+\frac{1}{8^{2}}+\ldots\right),
\end{aligned}
$$

de donde se tiene la tesis.

Proposición 3.1 ((Euler)). Existe un número infinito de números primos.

\section{Demostración:}

Supongamos que existiera un número finito de números primos, $2,3,5, \ldots, p$ con $p$ el mas grande número primo.

Sabemos que $\sum a^{n}=\frac{1}{1-a}$ si $|a|<1$, luego se tiene

$$
\begin{aligned}
& 1+\frac{1}{2}+\frac{1}{2^{2}}+\frac{1}{2^{3}}+\frac{1}{2^{4}}+\ldots=\frac{1}{1-\frac{1}{2}}=2 \\
& 1+\frac{1}{3}+\frac{1}{3^{2}}+\frac{1}{3^{3}}+\frac{1}{3^{4}}+\ldots=\frac{1}{1-\frac{1}{3}}=\frac{3}{2} \\
& 1+\frac{1}{5}+\frac{1}{5^{2}}+\frac{1}{5^{3}}+\frac{1}{5^{4}}+\ldots=\frac{1}{1-\frac{1}{5}}=\frac{5}{4} \\
& 1+\frac{1}{p}+\frac{1}{p^{2}}+\frac{1}{p^{3}}+\frac{1}{p^{4}}+\ldots=\frac{1}{1-\frac{1}{p}}=\frac{p}{p-1} .
\end{aligned}
$$

Ahora Euler considera el producto (remarcamos que este teorema fue probado por Euclides en "Los Elementos")

$$
\begin{aligned}
& \left(1+\frac{1}{2}+\frac{1}{2^{2}}+\frac{1}{2^{3}}+\frac{1}{2^{4}}+\ldots\right)\left(1+\frac{1}{3}+\frac{1}{3^{2}}+\frac{1}{3^{3}}+\frac{1}{3^{4}}+\ldots\right)\left(1+\frac{1}{5}+\frac{1}{5^{2}}+\frac{1}{5^{3}}+\frac{1}{5^{4}}+\ldots\right) \\
& \ldots\left(1+\frac{1}{p}+\frac{1}{p^{2}}+\frac{1}{p^{3}}+\frac{1}{p^{4}}+\ldots\right)=(2)\left(\frac{3}{2}\right)\left(\frac{5}{4}\right) \ldots\left(\frac{p}{p-1}\right)
\end{aligned}
$$

Como el producto del lado derecho es finito, el producto de las sumas infinitas es también finito.

Pero, este producto se puede escribir convenientemente a nuestro interés (encontrar una contradicción), así solo consideramos los sumandos que conviene explicitar y se omiten los infinitos otros, así tal producto es igual a $1+\frac{1}{2}+\frac{1}{3}+\frac{1}{4}+\frac{1}{5}+\frac{1}{6}+\frac{1}{7}+\frac{1}{8}+\ldots$, suma que es divergente $\left(\sum_{n} \frac{1}{n^{p}}\right.$ converge si y solo si $\left.p>1\right)$; así se obtiene una contradicción.

4. Siglo XIX. El Rigor del Cálculo.. El siglo XIX es una etapa gloriosa para la matemática, una edad de oro, del rigor. Se inicia con el trabajo de Fourier sobre la conducción del calor y que abriría nuevas teorías en el análisis moderno. Pero sus argumentos matemáticos, y de otros trabajos, inquietaron a 
matemáticos como Bernard Bolzano (1781-1848) quien inicia un movimiento para rigorizar al cálculo. él fue un precursor de la teoría de conjuntos y consideró al método matemático como el único camino para hallar la verdad científica. Por otro lado, por aquella época aún no estaba suficientemente consistente la noción de "función continua", pero Bolzano tuvo claro esta noción, solo le faltó usar los $\delta^{\prime} s$ y $\epsilon^{\prime} s$. Escribió su obra fundamental "Estudio sobre las Funciones", que recién fue encontrada en 1920 y publicada en 1932. Rescatemos a su teorema del valor intermedio. Bolzano también se interesó por las funciones discontinuas; abrió el camino a nuevos métodos para rigorizar al cálculo, ideas como la de "límite", idea esencial para construir al análisis matemático.

"Pero sería un serio error pensar que solo se puede encontrar certeza en las demostraciones geométricas o en el testimonio de los sentidos.

A. L. Cauchy.".

La contribución de Fourier fue notable por las polémicas que ella originaría, entre ellas la noción de función. En tal panorama llega Augustin L. Cauchy (1789-1857), quien publica en 1821 su notable libro "Curso de Análisis" en donde inicia la ansiada rigorización del cálculo infinitesimal; posteriormente, en 1823, expone la necesidad de enseñar el cálculo sobre la base de principios consistentes. El punto de partida es la noción de "límite", la que define en la forma:

“ «Cuando los sucesivos valores atribuidos a una variable se aproximan indefinidamente a un valor fijo, tanto que al final difieren de él tanto como uno desea, esta última cantidad es denominada el límite de todas las otras.»

En 1844 Cauchy considera la función

$$
y=\left\{\begin{array}{r}
x \ldots x \geq 0 \\
-x \ldots x<0
\end{array}\right.
$$

es decir $y=|x|$, función que es discontinua según Euler pues está definida por dos expresiones analíticas, pero Cauchy observa que

$$
|x|=\frac{2}{\pi} \int_{0}^{\infty} \frac{x^{2}}{t^{2}+x^{2}} d t
$$

y por lo tanto $|x|$ serie continua, según Euler; una contradicción. Por otro lado, aun con los defectos de algunos de sus resultados, sus trabajos sobre la convergencia de las series fueron muy novedosas para su época. Con Cauchy se inicia una nueva era de enfocar el cálculo infinitesimal surgiendo así lo que sería el análisis matemático.

4.1. La integral de Cauchy.. «Sea $f(x)$ una función continua definida sobre el intervalo $[a, b]$. La integral $\int_{a}^{b} f(x) d x$ es definida como el límite de las sumas

$$
f(a)\left(x_{1}-a\right)+f\left(x_{1}\right)\left(x_{2}-x_{1}\right)+\ldots+f\left(x_{n-1}\right)\left(x_{n}-x_{n-1}\right)
$$

donde se tiene la partición $a<x_{1}<x_{2}<\ldots<x_{n-1}<b$ de $[a, b]$, y el límite es tomado cuando el $\max \left(x_{i}-x_{i-1}\right)$ tiende a cero. $\gg$

Posteriormente, Cauchy extiende esta definición para el caso de funciones discontinuas con un número finito de discontinuidades. Aún más, Cauchy estudia a las integrales impropias definiendo la noción de "valor principal" de una integral. Sus integrales singulares, son precursoras de la "teoría de integrales singulares" de A. Calderón - A. Zygmund, cuya evolución condujo a las "integrales de Cauchy", área relativamente nueva.

La relación de la matemática con la física y la astronomía condujo a investigar nuevos tipos de funciones, sus límites e integrales. En este escenario surge el ilustre matemático Carl F. Gauss (1777 - 1855) quien fue muy exigente con el rigor matemático. Probó que si $a_{n} \rightarrow 0$, la serie $\sum_{n=1}^{\infty} a_{n} \cos n x$ es convergente. Al igual que Legendre, Abel y Jacobi, Gauss trabajó en la teoría de las funciones elípticas. Tuvo dos distinguidos discípulos Peter G. Lejeune - Dirichlet(1805-1859) y Bernhard Riemann (1826-1866) quienes contribuirán mucho en el análisis. Fourier había mostrado que una variedad de funciones pueden ser representadas por series trigonométricas pero una cuestión abierta era: "encontrar las condiciones precisas para que una función tenga una serie de Fourier convergente a la función”. Cauchy intentó clarificar este panorama pero no tuvo éxito. Dirichlet entra en escena. En 1829 da condiciones suficientes con tal objetivo; en ese trabajo introdujo las llamadas "integrales de Dirichlet"

$$
\int_{0}^{a} \frac{\operatorname{senn} x}{\operatorname{sen} x} f(x) d x y \int_{a}^{b} \frac{\operatorname{senn} x}{\operatorname{sen} x} f(x) d x, 0<a<b,
$$

así como construye la "función de Dirichlet" $f(x)=\left\{\begin{array}{c}c \ldots x \text { racional } \\ d \ldots x \text { irracional }\end{array}\right.$, función que no es R-integrable! 
Dirichlet conjetura que es posible definir la integral $\int_{a}^{b} f(x) d x$ para funciones $f(x)$ con un conjunto infinito de puntos de discontinuidad siempre que este conjunto fuera "pequeño", esto es, "si cualquiera que fueran los números $a<b$, existieran $r$ y $s$ tales que $a<r<s<b$ tal que $f(x)$ sea continua en el intervalo $[r, s]$ ". Observemos que la función de Dirichlet no es pequeña.

Dirichlet, en 1829, no logró dar una respuesta definitiva a la cuestión: ¿Cuándo una función es integrable? ... Cauchy considera funciones continuas, a lo máximo seccionalmente continuas. Riemann, brillante matemático, entra en acción. Al representar $f(x)$ por una serie trigonométrica tuvo necesidad de determinar los coeficientes

$[F] \int_{-\pi}^{\pi} f(x) \cos x d x \quad y \quad \int_{-\pi}^{\pi} f(x) \operatorname{sen} x d x$, pero ¿qué significa $\int_{a}^{b} f(x) d x$ ?; donde $f(x)$ es arbitraria.

¿Cuáles son las funciones que tienen integral?

Luego de investigar en este problema; Riemann llegó al criterio siguiente: "una función $f(x)$ es integrable si y solamente si

dado $\varepsilon>0$ arbitrario y $\sigma>0$, es posible hallar $\delta>0$ tal que para toda partición $P$ con $|P|<\delta$ la suma de las longitudes de los intervalos $\ell_{i}$, donde $w_{i} \geq \sigma$, es menor que $\varepsilon$ ".

$w_{i}$ representa la oscilación de $f$ es el intervalo $I_{i}$.

El trabajo de Riemann sobre series trigonométricas tuvo un gran impacto en el desarrollo del análisis de Fourier.

¿Toda serie trigonométrica es una serie de Fourier? (es decir, sus coeficientes son de la forma [F]); ¿la serie trigonométrica asociada a una función, es única? Riemann pudo haber clarificado estas cuestiones pero falleció en plena madurez. Menos mal que en este siglo XIX hubieron otros geniales matemáticos como Abel, K. Weierstrass, H. Heine, ..., que contribuyeron fuertemente en la consolidación del análisis. En particular las contribuciones de Heinrich Heine (1821-1881) quien resalta la importancia de la convergencia uniforme en la integración término a término de la serie. Formula el problema de la unicidad de la serie trigonométrica, cuestión que dio a un joven matemático.

Tal joven fue Georg Cantor (1845-1918) quien llegó a escribir cinco artículos sobre el problema de la unicidad (de la representación en serie trigonométrica) en el periodo 1870-1872. Cantor remueve la hipótesis de que la función $f(x)$ debe tener un conjunto finito de discontinuidades y llega a considerar conjuntos de puntos bastantes generales en la recta. Estas investigaciones le condujeron a la formulación de su famosa "teoría de conjuntos".

Veamos. Cantor luego de su graduación, se dedica al análisis real en la universidad de Halle donde Heine trabajaba en series de Fourier de la forma

$$
\frac{d_{o}}{2}+\sum_{n=1}^{\infty}\left(c_{n} \operatorname{sen} n x+d_{n} \cos n x\right), c_{n}, d_{n} \in \mathbb{R}
$$

Heine propuso el problema de la unicidad de las series de Fourier: "suponga que una serie de Fourier converja a una función dada en algún sentido, ¿puede haber otra serie de Fourier convergiendo a la misma función, en el mismo sentido? Cantor trabajo en este problema y llegó al siguiente resultado:

Cantor (1871). "Si una serie trigonométrica converge puntualmente a cero, con excepción de ciertos valores del argumento, entonces todos sus coeficientes se anulan".

Nota. Cantor precisa el significado de "ciertos valores". Con él también se clarifica la idea del infinito, que por siglos atormentó a los pensadores!

5. La Teoría de Conjuntos.. Nuestro objetivo es representar aspectos básicos de la teoría de conjuntos vía la representación decimal pues todo número en $[0,1]$ se puede representar por una serie especial. Seguimos de cerca al excelente libro de A. Devinatz, [2].

Proposición 5.1. Sea $m$ un número positivo mayor que 1, entonces la serie $\sum_{k=1}^{\infty} \frac{a_{k}}{m^{k}}$, donde $a_{k} \in[0, m-1]$, converge a un número en $[0,1]$.

Demostración: Se tiene

$$
0 \leq \sum_{k=1}^{\infty} \frac{a_{k}}{m^{k}} \leq\left(\text { desde que } 0 \leq \frac{a_{k}}{m^{k}} \leq \frac{m-1}{m^{k}}\right)(m-1) \sum_{k=1}^{\infty}\left(\frac{1}{m}\right)^{k}
$$

y esta serie es una serie geométrica y se tiene

$$
\sum_{k=1}^{\infty}\left(\frac{1}{m}\right)^{k}=\sum_{k=0}^{\infty}\left(\frac{1}{m}\right)^{k}-1=\frac{1}{1-\frac{1}{m}}=\frac{1}{m-1}
$$


De esta manera se obtiene $0 \leq \sum_{k=1}^{\infty} \frac{a_{k}}{m^{k}} \leq 1$. En conclusión, por el criterio de comparación se tiene que $\sum_{k=1}^{\infty} \frac{a_{k}}{m^{k}}$ es convergente y converge a un número en $[0,1]$.

El siguiente resultado es el inverso de la proposición 5.1 y completa lo que necesitamos para lograr el objetivo señalado antes.

Teorema 5.1. Sea $m$ un entero positivo mayor que 1, entonces para todo $\alpha \in[0,1]$ existe una sucesión $\left(a_{k}\right)$, con rango en $[0, m-1]$ tal que

\section{Demostración:}

$$
a=\sum_{k=1}^{\infty} \frac{a_{k}}{m^{k}} .
$$

Se comienza con el siguiente argumento:

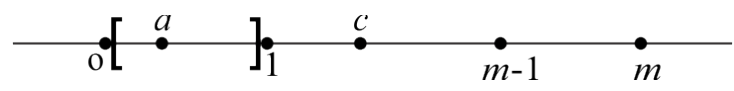

Figura 5.1: Representación de $a \in[0,1]$

Sea $a_{1}$ el mas grande entero en $[0, m-1]$ tal que $\frac{a_{1}}{m} \leq a$;

$a_{2}$ el mas grande entero en $[0, m-1]$ tal que $\frac{a_{1}}{m}+\frac{a_{2}}{m} \leq a$

$a_{n}$ el mas grande entero en $[0, m-1]$ tal que $\frac{a_{1}}{m}+\frac{a_{2}}{m^{2}}+\ldots+\frac{a_{n}}{m^{n}} \leq a$.

De esta manera se construye una sucesión $\left(a_{n}\right)$ tal que $\sum_{k=1}^{n} \frac{a_{k}}{m^{k}} \leq a .\left(^{*}\right)$

Así, por inducción, se verifica que existe una (única) sucesión $\left(a_{n}\right)$ tal que para todo $n \in \mathbb{N}$ se satisface la desigualdad (*); además, para toda $j \in[1, n]$ si $c \in[0, m-1]$ y $c>a_{j}$ se tendrá $\sum_{k \in A_{j}} \frac{a_{k}}{m^{k}}+\frac{c}{m^{j}}>a$, donde $A_{j}$ son los elementos de $[1, n]$ diferentes de $j$.

Luego, la sucesión $\left\{\frac{a_{1}}{m^{1}}+\frac{a_{2}}{m^{2}}+\ldots+\frac{a_{n}}{m^{n}}\right\}$ es monótona no-decreciente y acota superiormente por a, es decir, se tiene $\{\ldots\} \rightarrow b \leq a$. Se verá que $b=a$.

De esta manera, $\forall n \in \mathbb{N}$ se tiene: $\sum_{k=1}^{n} \frac{a_{k}}{m^{k}} \leq \sum_{k=1}^{\infty} \frac{a_{k}}{m^{k}}=b \leq a$.

Supongamos que $b<a$. Desde que $m>1, \frac{1}{m^{n}} \rightarrow 0$ si $n \rightarrow \infty$, existirá el más pequeño entero positivo $p$ tal que $\frac{1}{m^{p-1}} \leq a-b$. Ahora, si $n \geq p$ se tiene

$\frac{1}{m^{n-1}} \leq \frac{1}{m^{p-1}} \leq a-b$.

Luego, $\sum_{k=1}^{n-1} \frac{a_{k}}{m^{k}}+\frac{m-1}{m^{n}} \leq b+\frac{1-\frac{1}{m-1}}{m^{n-1}} \leq b+\frac{1}{m^{n-1}} \leq b+(a-b)=a$

Luego, por la forma de como la sucesión $\left(a_{k}\right)$ fue definida se deberá tener que $a_{n}=m-1$.

Sea ahora $q$ el mas pequeño entero tal que $n \geq q$ implica que $a_{n}=m-1$. Entonces $q>1$ pues si $q \leq 1$ se tendría usando la serie geométrica

$\left(\sum_{k=0}^{\infty}\left(r^{k}\right) \rightarrow \frac{1}{1-r}, s i|r|<1\right) b=\sum_{k=1}^{\infty} \frac{m-1}{m^{k}}=1 \geq a$, una contradicción pues estamos asumiendo que $b<a$.

Por otro lado, desde que

$\sum_{k=q}^{\infty} \frac{m-1}{m^{k}}=\sum_{k=q-1}^{\infty} \frac{m-1}{m^{k}}-\frac{m-1}{m^{q-1}}=\frac{m}{m^{q-1}}-\frac{m-1}{m^{q-1}}=\frac{1}{m^{q-1}}$

se tiene que:

$b=\sum_{k=1}^{q-1} \frac{a_{k}}{m^{k}}+\frac{1}{m^{q-1}}=\sum_{k=1}^{q-2} \frac{a_{k}}{m^{k}}+\frac{a_{q-1+1}}{m^{q-1}}<a$. 
Pero, $a_{q-1}<m-1$, luego $a_{q-1}+1 \leq m-1$ lo cual es una contradicción desde que por construcción de $\left(a_{k}\right)$ no se permite esta desigualdad. Luego, $b=a$.

Observación 5.1. Si a $\in[0,1]$ entonces su representación, expansión decimal, es de la forma a = $0, a_{1} a_{2} a_{3} \ldots$, donde la base $m$ debe ser especificado; además, tal representación no es única en cierta situación, como es el caso de los números racionales. En el caso general de los números reales se tiene lo siguiente:

"Todo número real en $[0,1]$, excepto para un "decimal terminante” (como los números racionales, es decir, donde $a_{k}=0$ para $k$ suficientemente grande), tiene una representación única en serie con respecto a un número fijo $m \in \mathbb{N}$, $m>1$.

Además, un decimal terminante puede ser representado en solo dos formas: ( $i$ ) en una serie finita ó (ii) en una serie donde existe $n$ tal que $\forall k \geq n$, se tiene $a_{k}=n-1 . "$

Sabemos que el conjunto de los números reales no es enumerable; ahora se usará las series o representaciones decimales para probar tal resultado. Es suficiente probar el siguiente teorema.

Teorema 5.2. El intervalo $[0,1]$ no es enumerable.

Demostración:

Sabemos que el conjunto $Q$ de los racionales es enumerable y por tanto el conjunto de los decimales terminantes es enumerable (siendo un subconjunto infinito de $Q$ ) y de esta manera el conjunto de todos los números en $[0,1]$ no es finito; además, el conjunto de las sucesiones, cada una teniendo su rango en $[0, m-1]$, es enumerable o no enumerable si y solo si el conjunto de los números en $[0,1]$ es enumerable o no enumerable, respectivamente.

Bien, supongamos que el conjunto de las sucesiones, cada una teniendo su rango en $[0, m-1]$, fuera enumerable y sea $S$ este conjunto. De esta manera existiría una función $1-1$, sobre,

$$
\begin{aligned}
& f: \mathbb{N} \rightarrow S \\
& n \rightarrow f(n)=\left(a_{k}^{n}\right)
\end{aligned}
$$

donde $a_{k}^{n}$ es un entero en [0,m-1]. La idea ahora es definir la sucesión $\left(a_{k}\right)$ vía:

$$
a_{k}=\left\{\begin{array}{c}
a_{k}^{k}-1 \ldots a_{k}^{k} \neq 0 \\
m-1 \ldots a_{k}^{k}=0
\end{array},\right.
$$

donde remarcamos que esta sucesión existe pues $m \geq 2$. Además se observa que la sucesión $\left(a_{k}\right)$ tiene su rango en $[0, m-1]$ y por tanto existirá un $q \in \mathbb{N}$ tal que

$$
\left(a_{k}\right)=S(q)=\left(a_{k}^{q}\right) \text {. }
$$

esto es, $a_{k}=a_{k}^{q}, \forall k$; pero, si $k=p$ se tiene $a_{q} \neq a_{q}^{q}$ y así se obtiene una contradicción. Por lo tanto, $[0,1]$ no es enumerable.

\section{Ejemplo 5.1 (El Conjunto de Cantor).}

Si tomamos $m=3$ en las anteriores series se obtiene las llamadas "expansiones ternarias" de números en el intervalo $[0,1]$. Por definición, el conjunto de Cantor es el conjunto

$$
C=\left\{a \in[0,1] / a=\sum_{k=1}^{\infty} \frac{a_{k}}{3^{k}}, \text { donde } a_{k} \neq 1 \text { para todo } k \in \mathbb{N}\right\}
$$

El siguiente resultado resume las principales propiedades del conjunto C. ([2]).

Teorema 5.3. El conjunto de Cantor $C$ es un subconjunto de $[0,1]$ que es perfecto y no enumerable cuyo conjunto complemento $C_{c}$ consiste de la unión de intervalos abiertos disjuntos dos a dos, y la suma del total de las longitudes de estos intervalos es 1.

Demostración: La prueba se dará vía una sucesión de resultados parciales las que conducen a la tesis del teorema.

(i) $C$ es un conjunto cerrado.

En efecto: la idea es verificar que $C_{c}$ es un conjunto abierto.

Sea $b \in C_{c}$ y se verificará que existe un intervalo abierto $\left(\frac{p}{3^{j}}, \frac{p+1}{3^{j}}\right)$ tal que $b \in\left(\frac{p}{3^{j}}, \frac{p+1}{3^{j}}\right) \subset$ $C_{c} \cdot[\bullet]$

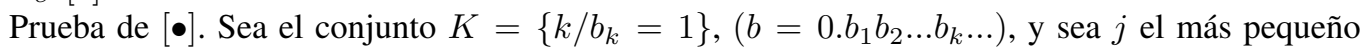
elemento en $K$ (así, $b_{j}=1$ ). Ahora, existe $k>j$ tal que $b_{k} \neq 2$ pues si $b_{k}=2$ para todo $k>j$, y desde que $\sum_{k=j+1}^{\infty} \frac{2}{3^{k}}=\frac{1}{3^{j}}(+)$, se tendría 


$$
b=\sum_{k=1}^{j-1} \frac{b_{k}}{3^{k}}+\frac{b_{j}}{3^{j}}+\sum_{k=j+1}^{\infty} \frac{2}{3^{k}}=\sum_{k=1}^{j-1} \frac{b_{k}}{3^{k}}+\frac{1}{3^{j}}+\frac{1}{3^{j}}=\left(\sum_{k=1}^{j-1} \frac{b_{k}}{3^{k}}+\frac{2}{3^{j}}\right) \in C,
$$

lo que es una contradicción pues $b \notin C$. De igual manera, existe $k>j$ tal que $b_{k} \neq 0$. En efecto, si $b_{k}=0$ para todo $k>j$, y usando (+), se tiene nuevamente

$b=\sum_{k=1}^{j-1} \frac{b_{k}}{3^{k}}+\frac{b_{j}}{3^{j}}+\sum_{k=j+1}^{\infty} \frac{b_{k}}{3^{k}}=\sum_{k=1}^{j-1} \frac{b_{k}}{3^{k}}+\frac{1}{3^{j}}=\left(\sum_{k=1}^{j-1} \frac{b_{k}}{3^{k}}+\sum_{k=j+1}^{\infty} \frac{2}{3^{k}}\right) \in C$,

y se tiene nuevamente una contradicción pues $b \in C_{c}$.

Ahora definamos $q$ vía: $\frac{q}{3^{j-1}}=\sum_{k=1}^{j-1} \frac{b_{k}}{3^{k}}$.

Bien, desde que $\forall k \in[1, j-1]$ implica que $b_{k}=0$ ó $b_{k}=2$ (por la definición de $j$ ) y de esta manera $b_{k}$ es par y así $q$ es par y $q<3^{j-1}$

(pues si $b_{k}=0, q=0 ;$ si $b_{k}=2,-\frac{q}{3^{j-1}}=2 \sum_{k=1}^{j-1} \frac{1}{3^{k}}<1$ ).

Entonces se tiene,

$$
\begin{aligned}
b & =\sum_{k=1}^{j-1} \frac{b_{k}}{3^{k}}+\frac{b_{j}}{3^{j}}+\sum_{k=j+1}^{\infty} \frac{b_{k}}{3^{k}}=\frac{q}{3^{j-1}}+\frac{1}{3^{j}}+\sum_{k=j+1}^{\infty} \frac{b_{k}}{3^{k}}<\frac{q}{3^{j-1}}+\frac{1}{3^{j}}+\sum_{k=j+1}^{\infty} \frac{2}{3^{k}} \\
& =\frac{q}{3^{j-1}}+\frac{1}{3^{j}}+\frac{1}{3^{j}}=\frac{q}{3^{j-1}}+\frac{2}{3^{j}} .
\end{aligned}
$$

También se tiene,

$$
b=\sum_{k=1}^{j-1} \frac{b_{k}}{3^{k}}+\frac{b_{j}}{3^{j}}+\sum_{k=j+1}^{\infty} \frac{b_{k}}{3^{k}}>\left(\text { desde que } b_{k}>0\right)>\sum_{k=1}^{j-1} \frac{b_{k}}{3^{k}}+\frac{1}{3^{j}}=\frac{q}{3^{j-1}}+\frac{1}{3^{j}} .
$$

De esta manera se ha probado que: $\frac{q}{3^{j-1}}+\frac{1}{3^{j}}<b<\frac{q}{3^{j-1}}+\frac{2}{3^{j}}$,

expresiones equivalentes a $\frac{3 q+1}{3^{j}}<b<\frac{(3 q+1)+1}{3^{j}}$; si ponemos $p=3 q+1$, esta expresión nos dice que todo $b \in C_{c}$ está en un intervalo de la forma $\left(\frac{p}{3^{j}}, \frac{p+1}{3^{j}}\right)$, donde $p$ es un número impar y además, $1>b>\frac{p}{3^{j}}$ ó $p<3^{j}$.

Ahora se postula que: $\left(\frac{p}{3^{j}}, \frac{p+1}{3^{j}}\right) \subset C_{c}$.

En efecto, observamos que $p=3 q+1=3\left(3^{j-1} \sum_{k=1}^{j-1} \frac{b_{k}}{3^{k}}\right)+1=3^{j} \sum_{k=1}^{j-1} \frac{b_{k}}{3^{k}}+1$

$=3^{j} \sum_{k=1}^{j-1} \frac{b_{k}}{3^{k}}+\frac{3^{j}}{3^{j}}=3^{j}\left(\sum_{k=1}^{j-1} \frac{b_{k}}{3^{k}}+\frac{1}{3^{j}}\right)$. Por tanto,

$\frac{p}{3^{j}}=\sum_{k=1}^{j-1} \frac{b_{k}}{3^{k}}+\frac{1}{3^{j}}=\sum_{k=1}^{j} \frac{b_{k}}{3^{k}}$. Escribamos $\frac{p}{3^{j}}=\sum_{k=1}^{j} \frac{p_{k}}{3^{k}}, p_{k} \in[0,2]$.

Como $p$ es impar, existirá $k \in[1, j]$ tal que $p_{k}=1$ (pues si $p_{k} \neq 1$ para todo $k \in[1, j]$ se tendría $p_{k}=0$ ó $p_{k}=2$, y entonces $p$ sería par, contradicción).

Ahora supongamos que " $a$ " satisface $\frac{p}{3^{j}}<a<\frac{p+1}{3^{j}} \quad$ y $\quad a=\sum_{k=1}^{\infty} \frac{a_{k}}{3^{k}}$.

Entonces se tiene $a_{k}=p_{k}, \quad \forall k \in[1, j]$.

En efecto, razonemos por el absurdo.

Sea el conjunto $X=\left\{k / k \in[1, j]\right.$ y $\left.a_{k} \neq p_{k}\right\}$, y sea $r$ el más pequeño entero en $X$. Luego existen dos posibilidades: $a_{r}>p_{r}$ ó $a_{r}<p_{r}$.

- Supongamos que $a_{r}>p_{r}$ ó $a_{r} \geq p_{r}+1$, entonces

$$
a=\sum_{k=1}^{\infty} \frac{a_{k}}{3^{k}} \geq \sum_{k=1}^{r} \frac{a_{k}}{3^{k}} \geq \sum_{k=1}^{r} \frac{p_{k}}{3^{k}}+\frac{1}{3^{r}} \geq \sum_{k=1}^{r} \frac{p_{k}}{3^{k}}+\sum_{k=r+1}^{\infty} \frac{2}{3^{k}} \geq \sum_{k=1}^{j} \frac{p_{k}}{3^{k}}+\sum_{k=j+1}^{\infty} \frac{2}{3^{k}}=\frac{p}{3^{j}}+\frac{1}{3^{j}}=\frac{p+1}{3^{j}}
$$

lo cual es una contradicción con $a<\frac{p+1}{3^{j}}$. 
Supongamos ahora que $a_{r}<p_{r}$ ó $a_{r} \leq p_{k}-1$. Entonces se tendrá

$$
a=\sum_{k=1}^{\infty} \frac{a_{k}}{3^{k}}=\sum_{k=1}^{r} \frac{a_{r}}{3^{k}}+\sum_{k=r+1}^{\infty} \frac{a_{k}}{3^{k}} \leq \sum_{k=1}^{r} \frac{p_{k}}{3^{k}}-\frac{1}{3^{r}}+\sum_{k=r+1}^{\infty} \frac{2}{3^{k}}=\sum_{k=1}^{r} \frac{p_{k}}{3^{k}} \leq \sum_{k=1}^{j} \frac{p_{k}}{3^{k}}=\frac{p}{3^{j}},
$$

lo cual aun es una contradicción pues $\frac{p}{3^{j}}<a$.

Así se ha probado que $a \in C_{c}$ pues existe $k \in[1, j]$ tal que $a_{k}=p_{k}=1$. De esta manera, si $b \in C_{c}$, existe $\left(\frac{p}{3^{j}}, \frac{p+1}{3^{j}}\right)$ tal que $b \in\left(\frac{p}{3^{j}}, \frac{p+1}{3^{j}}\right) \subset C_{c}$, luego $C_{c}$ es un conjunto abierto y por tanto el conjunto de Cantor $C$ es cerrado. (i) $\square$

Observación 5.2. Los anteriores argumentos nos permite afirmar que, desde que

$\frac{q}{3^{j-1}}+\frac{1}{3^{j}}<b<\frac{q}{3^{j-1}}+\frac{2}{3^{j}}$, se tenga $C_{c}=\cup I_{q, n}$, donde

$I_{q, n}=\left(\frac{q}{3^{n-1}}+\frac{1}{3^{n}}, \frac{q}{3^{n-1}}+\frac{2}{3^{n}}\right)$, donde $\frac{q}{3^{n-1}}=\sum_{k=1}^{n-1} \frac{q_{k}}{3^{k}}$, con $q_{k} \in\{0,2\}$.

Además, tales intervalos $I_{q, n}$ son disjuntos dos a dos, esto es, si $(p, k) \neq(q, n)$ entonces $I_{p, k} \cap$ $I_{q, n}=\phi$.

En efecto, por el absurdo, supongamos que $a \in I_{p, k} \cap I_{q, n}$, entonces $a=\sum_{j=1}^{n-1} \frac{q_{j}}{3^{j}}+\frac{1}{3^{n}}+$ $\sum_{j=n+1}^{\infty} \frac{a_{j}}{3^{j}}=($ unicidad de la representación $) \sum_{j=1}^{k-1} \frac{p_{j}}{3^{j}}+\frac{1}{3^{k}}+\sum_{j=k+1}^{\infty} \frac{a_{j}}{3^{j}} .(*)$ Ahora,

- Si $k<n$, vía (*), se observa que $q_{k}=1$, lo cual es contradicción con $q_{k} \in\{0,2\}$;

- Si $k=n$, entonces $p_{i}=q_{j}$ para $j \in[1, n-1](\operatorname{ver}(*))$,contradicción pues $p \neq q$ por construcción de $q$.

- Si $k>n$, ó $n<k$ se obtiene aun una contradicción (intercambiando el papel de k y $\mathrm{n}$ ).

De esta manera se tiene $I_{p, k} \cap I_{q, n}=\phi$ para $(p, k) \neq(q, n)$.

(ii) . De $C_{c}=\cup I_{q, n}$, se obtiene que la suma de las longitudes de los intervalos $I_{q, n}^{\prime} s$ es igual a $\sum_{n=1}^{\infty} \frac{2^{n-1}}{3^{n}}=1$

En efecto, para $n$ fijo y observando que $q_{k} \in\{0,2\}$, de $q=3^{n-1} \sum_{k=1}^{n-1} \frac{q_{k}}{3^{k}}$ se tiene que existen $2^{n-1}$ veces q's, esto es, existen exactamente $2^{n-1}$ intervalos de la forma $I_{q, n}$ y cada uno de estos intervalos tienen una longitud igual a $\frac{1}{3^{n}}$.

Recordando que $C_{c}=\cup I_{q, n}$, y que estos intervalos son disjuntos dos a dos, la longitud (ó "medida") de $C_{c}$ es dado por

$$
\sum_{n=1}^{\infty} \frac{2^{n-1}}{3^{n}}=1
$$

Corolario. Desde que $C \cup C_{c}=[0,1]$ y $\left|C_{c}\right|=1$ (medida de $C_{c}$ ), se tiene que la medida del conjunto de Cantor $C$ es cero, esto es, $|C|=0$ !

(iii) El Conjunto de Cantor $C$ es un Conjunto Perfecto.

Un conjunto es llamado perfecto si es cerrado y todo punto del conjunto es un punto de acumulación del conjunto (Recordemos que un número real $a$ es un punto de acumulación de un conjunto $A$ si $\forall \varepsilon>0, \exists x \in A, x \neq a$, tal que $|x-a|<\varepsilon)$.

De esta manera la tesis a verificar es que todo punto de $C$ es un punto de acumulación de $C$.

En efecto, sea $a \in C$; un caso es que $a=\sum_{k=1}^{\infty} \frac{a_{k}}{3^{k}}$, con $a_{k} \in\{0,2\}$, y donde $a_{k} \neq 0$ para infinitos números $k^{\prime} s$; de esta manera, las sumas parciales de esta serie son todas diferentes de $a$, ellas están en $C$ (ver definición de $C$ ), y convergen a $a$. Luego $a$ es un punto de acumulación de $C$. En el otro caso, si se tuviera $a=\sum_{k=1}^{j} \frac{a_{k}}{3^{k}}$, con $a_{k} \in\{0,2\}$, entonces para todo $n>j$ se construye $a_{n}=a+\frac{2}{3^{n}}$ y se tiene: $a \in C, a_{n} \neq a$ y $a_{n} \rightarrow a$. Luego $a$ es un punto de acumulación de $C$. Como $C$ es un conjunto cerrado y todo $a \in C$ es un punto de acumulación, se tiene que $C$ es un 
conjunto perfecto.

(iv) El Conjunto de Cantor $C$ no es enumerable.

En este caso, expansión decimal, la prueba de la no-enume rabilidad de $C$ hace uso también del "método de la diagonal" de Cantor. Bien, nuevamente supongamos que $C$ fuera un conjunto enumerable y por tanto existiría una función 1-1, definida apropiadamente para lograr la tesis; así se define la función 1-1.

$$
\begin{aligned}
f: \mathbb{N} & \rightarrow C \\
k & \rightarrow f(k)=a^{k}
\end{aligned}
$$

La idea es considerar la sucesión $\left(a_{k}\right)$, donde

$$
a_{k}=\left\{\begin{array}{r}
0 \ldots a_{k}^{k}=2 \\
2 \ldots a_{k}^{k}=0
\end{array}\right.
$$

(Observemos que se usa 0 y 2 al definir $a_{k}$, desde que $a^{k}=\left(a_{n}^{k}\right) \in C$ y $a_{n}^{k} \in\{0,2\}$.

Ahora la idea es determinar un número en base a la sucesión $\left(a_{k}\right)$, en su expresión ternaria, número que estaría en el conjunto $C$ pero no estaría en el rango de $f$ (por la definición de $f$ y de $a_{k}$ ).

Por tanto de (i) - (iv) se tiene probado el teorema 3.

Nota. ¿Cómo se describe $C$ geometricamente en el intervalo $[0,1]$ ? ...

Recordemos que $I_{q, n}=\left(\frac{q}{3^{n-1}}+\frac{1}{3^{n}}, \frac{q}{3^{n-1}}+\frac{2}{3^{n}}\right)$, donde $q=3^{n-1} \sum_{k=1}^{n-1} \frac{q_{k}}{3^{k}}$, con $q \in\{0,2\}$; vimos que $q$ es un número par y que $q<3^{n-1}$. Así, en particular se tiene $I_{0,1}=\left(\frac{1}{3}, \frac{2}{3}\right) \ldots$ es el tercio intervalo central de $[0,1]$.

$I_{0,2}=\left(\frac{1}{9}, \frac{2}{9}\right)$ y $I_{2,2}=\left(\frac{7}{9}, \frac{8}{9}\right) \ldots$ estos intervalos son los "tercios intervalos centrales" de los intervalos que quedan después de haber retirado $I_{0,1}$.

Y así continuamos con esta metodología, es decir, retirando los "tercios intervalos centrales" de los intervalos que quedan después de cualquier etapa, un número enumerable de etapas.

Y así, lo que queda en $[0,1]$ es ... el conjunto de Cantor $C$.

Pareciera que casi no queda nada! ... pero el teorema 3 nos dice que $C$ es un conjunto con propiedades interesantes, entre otras.

ORCID and License

Alejandro Ortiz Fernández https: / / orcid.org/0000-0002-9380-4301

This work is licensed under the Creative Commons - Attribution 4.0 International (CC BY 4.0)

\section{Referencias}

[1] Delachet A. Análisis Matemático. Madrid: Edit. Tecnos. S.A. 1973.

[2] Devinatz A. Advanced Calculus. New York: Holt, Rinehartand Winston. 1968.

[3] Dunham W. El Universo de las Matemáticas. Madrid: Edic. Pirámide. 1995.

[4] Heath T L. The Works of Archimedes. Cambridge: Cambridge University Press. 1897.

[5] Jahnke H N. A History of Analysis. Essen: University of Essen, 2003.

[6] Ortiz A. Algunas Ideas sobre la Génesis del Cálculo. Infinitesimal. Selecciones Matemática. 2021; vol 8(1):197-218.

[7] Ortiz, A. La Matemática a Través de Clásicas áreas.[Internet] Lima: Vol. 2. PUCP. 2014. urlhttps://www.pucp.edu.pe/profesor/ jesus-ortiz-fernandez/publicaciones/?x\& pagina=1

[8] Stahl S. Real Analysis. A Historical Approach. New York: John Willey-Sons. 1999.

[9] Stillwell J. Mathematics and his History. New York: Springer. 2001. 